\title{
Infrared dark clouds from the ISOGAL survey*
}

\section{Constraints on the interstellar extinction curve}

\author{
P. Hennebelle ${ }^{1}$, M. Pérault ${ }^{1}$, D. Teyssier ${ }^{1}$, and S. Ganesh ${ }^{2}$ \\ 1 Laboratoire de Radioastronomie Millimétrique, UMR 8540 du CNRS, École Normale Supérieure et Observatoire \\ de Paris, 24 rue Lhomond, 75231 Paris Cedex 05, France \\ 2 Physical Research Laboratory, Navarangpura, Ahmedabad 380009, India
}

Received 28 July 2000 / Accepted 24 October 2000

\begin{abstract}
The ISO galactic survey provides images of the inner disk in two broad filters (around 7 and $15 \mu \mathrm{m}$ ) over some 15 square degrees, away from the brightest star forming regions. A multiresolution analysis of the images leads to a catalogue of infrared dark clouds, most of which are condensed cores of large molecular clouds, several $\mathrm{kpc}$ away from the Sun, seen in absorption in front of the diffuse galactic emission. The longitude distributions of the background emission and of the dark clouds correlate with known tracers of young population components. We analyse the morphology of the dark clouds and the intensity fluctuations within the cloud boundaries at the two wavelengths. The 7 to $15 \mu \mathrm{m}$ contrast ratio is $0.75 \pm 0.15$ for the clouds located away from the Galactic Centre $\left(|l|>1^{\circ}\right)$ and $1.05 \pm 0.15$ for the clouds closest to the Galactic Centre $\left(|l|<1^{\circ},|b|<0.2^{\circ}\right)$. Using a simple absorption model, we derive a 7 to $15 \mu \mathrm{m}$ opacity ratio equal to $0.7 \pm 0.1$ for the clouds located away from the Galactic Centre and estimate the opacity, $\tau$, of a few objects at $15 \mu \mathrm{m}$ in the range 1 to 4 . Several explanations for the variation of the contrast ratio, including absorption along the line of sight and local variations of the extinction curve are discussed.
\end{abstract}

Key words. ISM: clouds, dust, structure - Galaxy: structure - infrared: general

\section{Introduction}

Much progress has been made since the first detection of diffuse mid-infrared emission from the Galaxy by the AFGL rocket-borne survey of the infrared sky (Price 1981). IRAS provided remarkable allsky images around 12 and $25 \mu \mathrm{m}$. Following studies established that the mid-infrared emission from the solar neighbourhood was dominated by diffuse emission, namely re-radiated stellar light from solid particles and aggregates (Boulanger \& Pérault 1988). These results were extended to galactic scale (Pérault 1987). The mid- to far-IR colour modulations due to the respective contributions of equilibrium and non-equilibrium thermal emission by dust were emphasized, and evidence was given of interstellar extinction in the most central regions at $12 \mu \mathrm{m}$, a result not inconsistent with standard extinction curves and estimated

Send offprint requests to: M. Pérault,

e-mail: perault@lra.ens.fr

* Based on observations with ISO, an ESA project with instruments funded by ESA Member States (especially the PI countries: France, Germany, The Netherlands and the UK) and with the participation of ISAS and NASA. The images have been obtained and processed by the ISOGAL collaboration. line-of-sight column densities. The quality of the midinfrared images, limited by the accuracy of zodiacal emission modelling, unfortunately restricted all published IRAS galactic studies (e.g. Sodroski et al. 1989, 1994; Bloemen et al. 1990) to the 60 and $100 \mu \mathrm{m}$ bands. Fortunately enough, COBE/DIRBE succeeded (under other things) in producing an accurate zodiacal emission model (Kelsall et al. 1998), and sensitive galactic emission measurements at low angular resolution, which however, as far as we know, have not been much exploited at midinfrared wavelengths yet. Median resolution spectroscopy on the other hand, especially from the ISO short wavelength spectrometer, has provided a spectacular new insight into physico-chemical properties of dust and gas, at least close to bright enough sources.

It turns out though that the mid-infrared wavelength range has not been exhausted, and should still provide distinguished contributions to studies of galactic diffuse components (large scale distribution and properties of interstellar phases, disentanglement of stellar distributions from absorption biases), as well as to studies of stellar formation at galactic scale (detection of young stellar populations throughout the disk, heating budget for the interstellar components). As a matter of fact, the contents of 


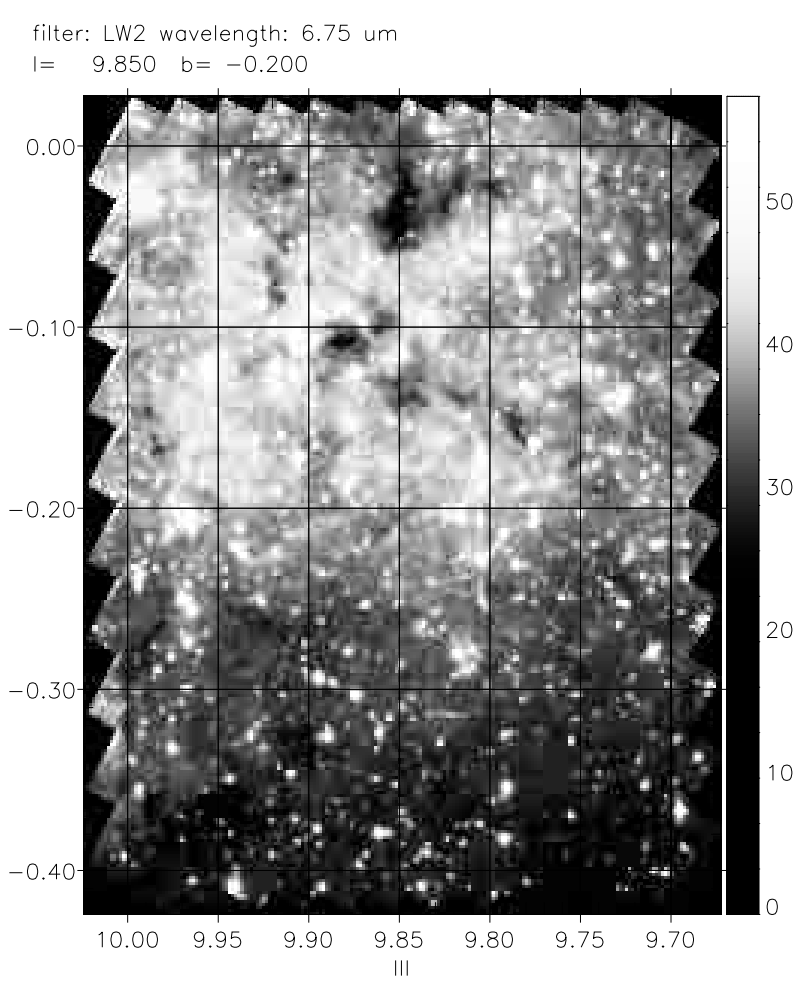

Fig. 1. 9.85-0.20 field of the ISOGAL survey at $7 \mu \mathrm{m}$. The intensity scale is in $\mathrm{MJy} \mathrm{sr}^{-1}$. Positions are in galactic coordinates

giant molecular clouds, for instance the gas fraction and temperature as a function of density, the density and pressure gradients in the condensation fronts at various spatial scales, and even worse, the links of their internal structure to their kinematics and dynamics, are still poorly documented.

The study of opaque condensed structures in a reasonably transparent environment is a long-used approach of interstellar structures (Barnard 1919; Bok 1947; Chandrasekhar \& Münch 1952; Lynds 1962, ...). ISO opened a similar opportunity in the mid-infrared on galactic scales, with sub-parsec resolution (Pérault et al. 1996) and detected many dark clouds in the inner Galaxy. Shortly later the Mid-course space experiment's (MSX) infrared imager surveyed the entire Galaxy, with a somewhat lower sensitivity and angular resolution than the ISO camera, and detected some 2000 infrared dark clouds mostly located between $-30^{\circ}$ and $+30^{\circ}$ which Egan et al. (1998) consider a new population of cold dense isolated clouds. Using $\mathrm{H}_{2} \mathrm{CO}$ observations, Carey et al. (1998) inferred column densities between $10^{23}$ and $10^{25} \mathrm{~cm}^{-2}$ and densities around $10^{5} \mathrm{~cm}^{-3}$.

The present paper reports the first outcome of a systematic search of deep absorption features in the 7 and $15 \mu \mathrm{m}$ images of the ISO galactic (scarse) survey (ISOGAL: Omont et al. 1999). A companion paper (Teyssier et al. 2001) will present spectroscopic followup observations conducted at the IRAM 30-m telescope on several dark objects. It includes intensive observations of millimeter lines of ${ }^{13} \mathrm{CO}, \mathrm{C}^{18} \mathrm{O}, \mathrm{HCN}, \mathrm{HNC}, \mathrm{HCO}^{+}$,

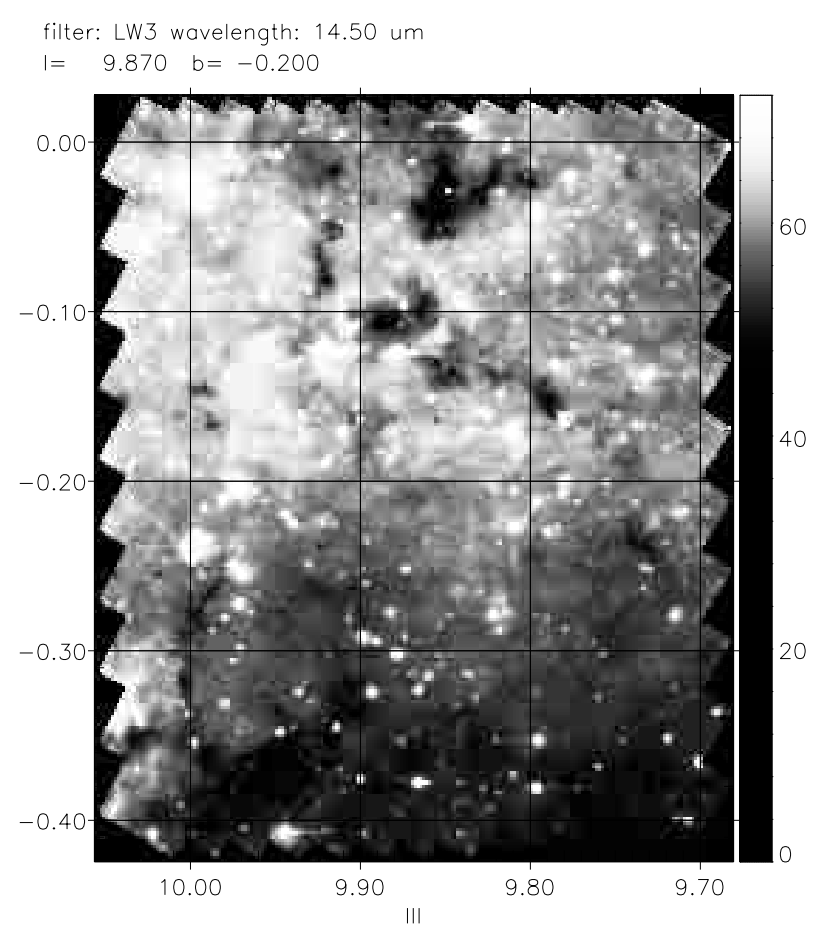

Fig. 2. Same field as Fig. 1 at $15 \mu \mathrm{m}$

$\mathrm{CH}_{3} \mathrm{C}_{2} \mathrm{H}, \mathrm{HC}_{3} \mathrm{~N}$, and $1 \mathrm{~mm}$ continuum emission. A subsequent study will focus on a systematic correlation between the infrared dark clouds and the stellar deficit in DENIS (near infrared) counts.

Section 2 briefly introduces the data and comments on the variations of the galactic mid-infrared background with longitude at 7 and $15 \mu \mathrm{m}$. In Sect. 3 we describe the systematic extraction of the dark features with the help of a multiscale analysis of the ISOGAL images. The objects detected at 2 wavelengths are cross-identified, and their morphology and distribution are then studied. The intensity fluctuations in the dark objects are statistically analysed in Sect. 4 and the mean 7 to $15 \mu \mathrm{m}$ opacity ratio is estimated. A few individual clouds are further examined in Sect. 5, leading to direct estimates of the optical depths. The study of the clouds located near the Galactic Centre and observed with narrower filters is presented in Sect. 6 . We then conclude in Sect. 7.

\section{The ISOGAL data}

Three different filters, LW2 (5-8.5), LW6 (7-8.5), and LW5 (6.5-7) were used at $7 \mu \mathrm{m}$ and two filters LW3 (12-18) and LW9 (14-16) around $15 \mu \mathrm{m}$. The ISOGAL survey covers about 18 square degrees mainly in the inner Milky-Way around 7 and $15 \mu \mathrm{m}$. The distribution is as follows:

- The inner Galaxy $\left(1.5^{\circ}<|l|<35^{\circ}\right)$ has been observed with the LW2 and LW3 filters (8.5 square degrees);

- The "outer" Galaxy $\left(|l|>35^{\circ}\right)$ has been observed with the LW2 and LW3 filters (3.5 square degrees);

- The central region $\left(|l|<1.5^{\circ}\right)$ has been observed with the LW6 and LW9 filters (2.5 square degrees); 

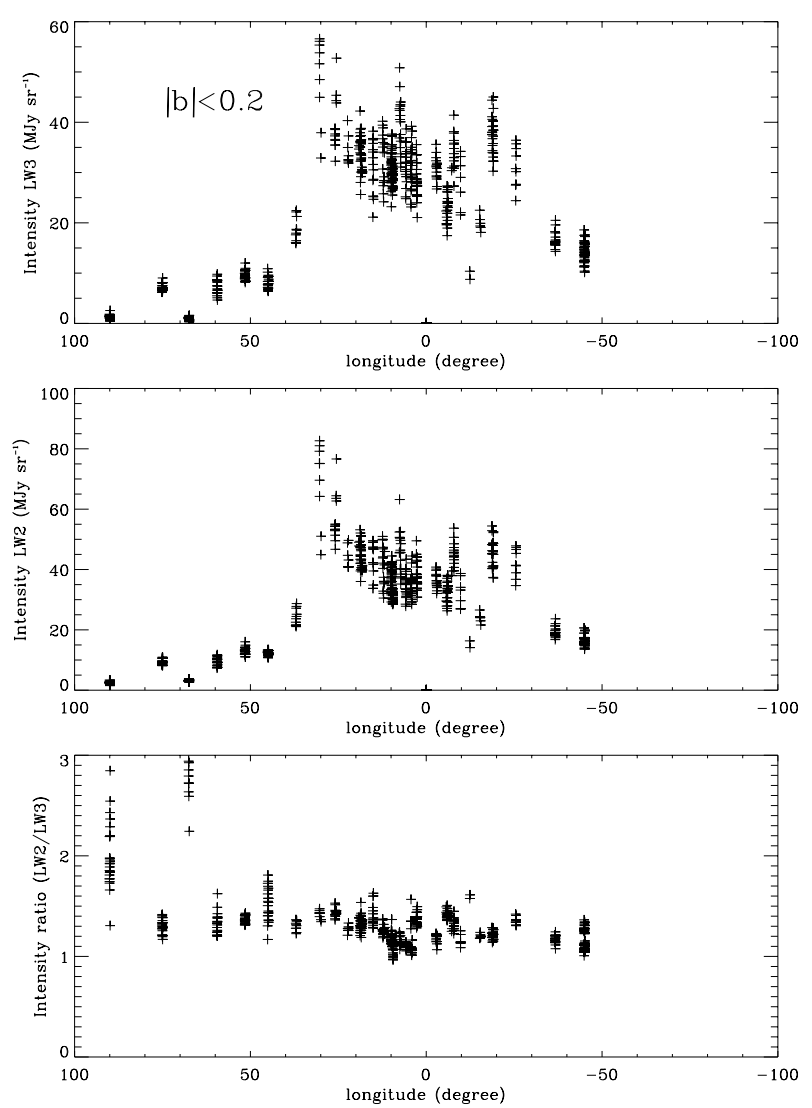

Fig. 3. Median intensity evaluated in square boxes of a $3 \cdot 5^{\prime} \times$ $3.5^{\prime}$ area as a function of longitude for $|b|<0.2^{\circ}$. Intensities in LW2 and LW3 and their ratio are displayed

- or, for the brightest, but still observable areas, with the LW5 and LW9 filters (1 square degree).

ISOGAL data were processed with the ISOCAM Interactive Analysis Package (Starck et al. 1999) including field distorsion correction and a preliminary correction of detector transients (Abergel et al. 1996). The zodiacal emission has been subtracted using the model of Kelsall et al. (1998). Comparing observations of the same region at different solar elongations, we estimated the uncertainty in the zodiacal background subtraction at $15 \mu \mathrm{m}$ to be better than $1 \mathrm{MJy} \mathrm{sr}^{-1}$.

Figures 1 and 2 are two ISOGAL images of the same field at $7 \mu \mathrm{m}$ (LW2) and $15 \mu \mathrm{m}$ (LW3). Both prominent emission and absorption features can be seen, as presented in earlier ISOGAL papers (e.g. Pérault et al. 1996), bringing deep insight into the structure of the interstellar medium, throughout the Galactic Plane. Several dark features can be seen. One of them $\left(\left(l=9.85^{\circ}, b=-0.05^{\circ}\right)\right.$, $\mathrm{DF}+9.85-0.05)$ is studied in detail (Sect. 5).

Figures 3 and 4 show the median filtered intensity (in MJy sr $\left.{ }^{-1}\right)$ of several lines of sight at low latitude $(|b|<$ $\left.0.2^{\circ}\right)$ and higher latitude $\left(0.2^{\circ}<|b|<0.4^{\circ}\right)$ respectively, as a function of longitude in the LW2 and LW3 filters. The intensity ratio is also shown. The median is computed in square boxes of a $3.5^{\prime} \times 3.5^{\prime}$ area. The intensities vary in a similar way as the IRAS sky brightness, away from bright
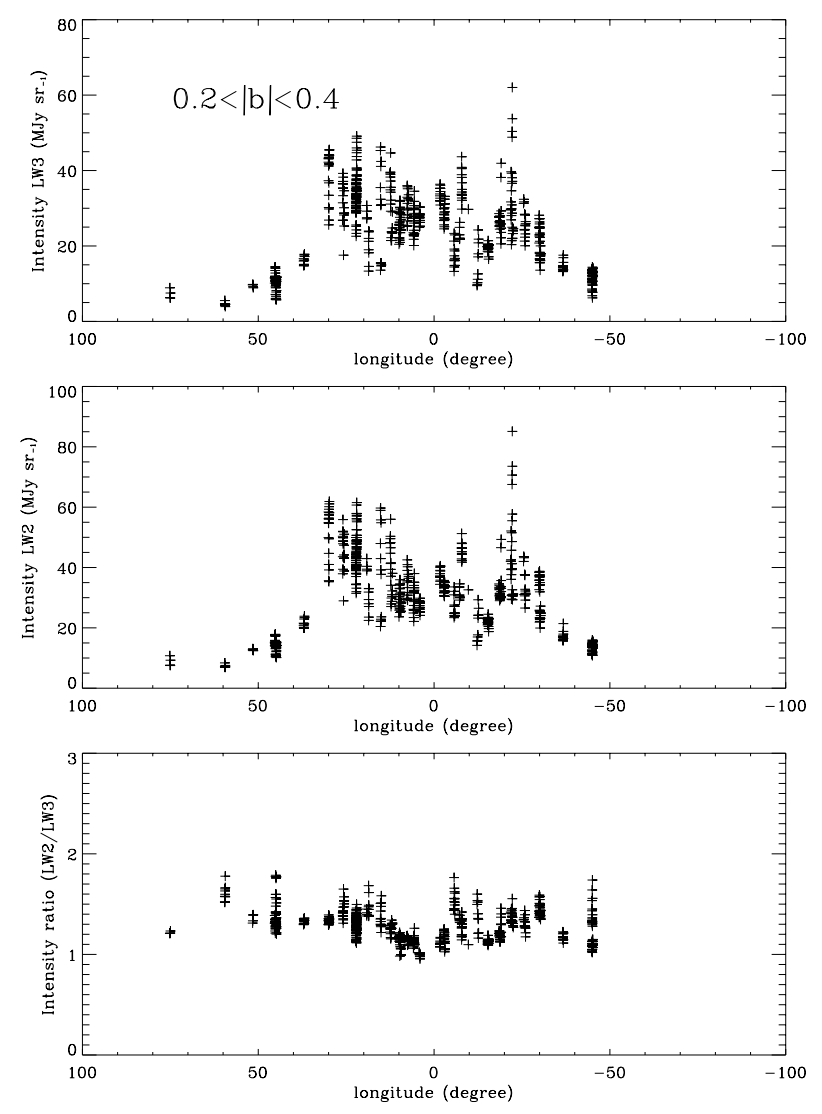

Fig. 4. Same as Fig. 3 for $0.2^{\circ}<|b|<0.4^{\circ}$

star forming regions. The ratio between the two bands is strikingly uniform: $1.2 \pm 0.2$ for all directions of not too low brightness (where the uncertainty dominates).

\section{Catalogue of dark absorption features}

In this section, we present a systematic detection of the dark objects seen in the ISOGAL images and statistically study some of their properties.

\subsection{Extraction of dark patterns from the images}

As illustrated in Figs. 1 and 2, the structures seen in absorption present various spatial scales, a complex morphology and various absorption contrasts. Galactic latitude gradient and diffuse emission structures produce significant variations (factor $\simeq 2$ ) in the background, at the scale of an individual image (0.1-0.5 degree). Point sources or extended sources add up to this contrasted background and to the inherent complexity of these structures, preventing simple algorithms from properly extracting the dark structures. Although sophisticated multiscale analysis tools are appropriate for complex extraction, they are not designed for images with both positive and negative structures. The following strategy was thus adopted:

- clipping of all positive structures to a local background estimate obtained with a median filter $(1 \times 0.5$ square arcmin); 


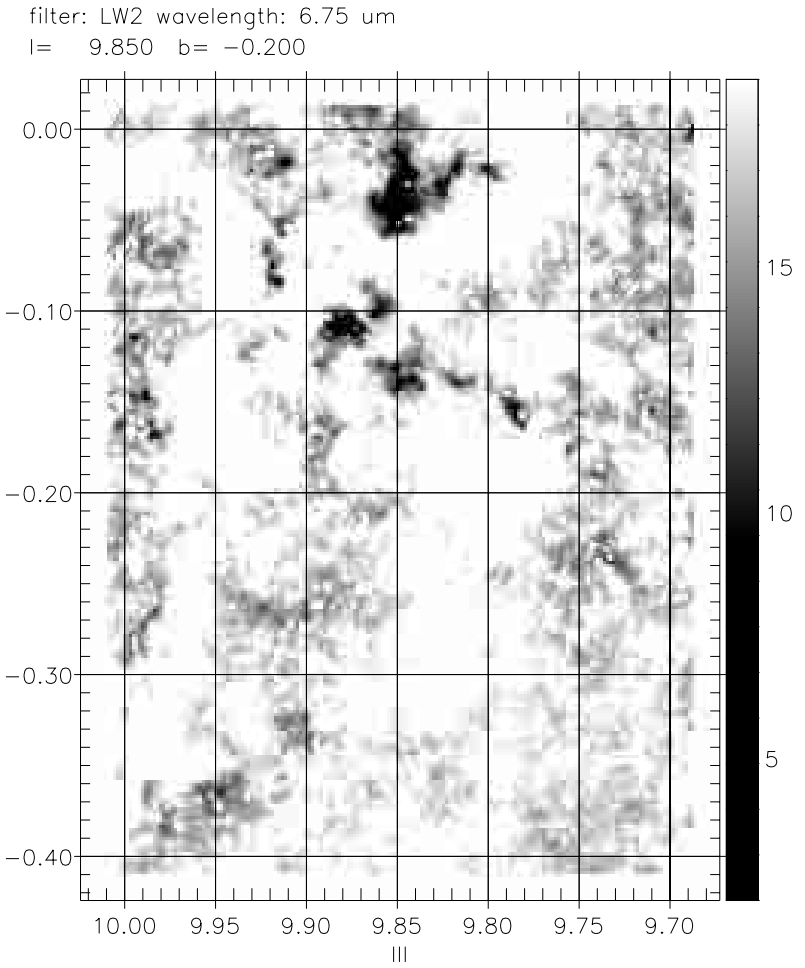

Fig. 5. $7 \mu \mathrm{m}$ LW2 image of the 9.85-0.20 field after clipping of all positive structures

- multi-scale (wavelet) analysis of the negative structures using the MVM package;

- object selection according to a minimal contrast criterion;

- cross identification of the 7 and $15 \mu \mathrm{m}$ lists.

The Multi-scale Vision Model (MVM) consists of the following steps (Rué \& Bijaoui 1997):

- wavelet transform;

- thresholding in the wavelet transform space, (the noise is assumed to be a Gaussian white noise, the threshold is set to $2.5 \sigma$ );

- scale by scale segmentation;

- interscale connectivity;

- objects identification;

- reconstruction.

The wavelet retained is a linear B-spline. In order to avoid non-significant objects too close from the pixel size or from the scale of the image $p\left(2^{p} \leq S_{\mathrm{im}} \leq 2^{p+1}\right.$, where $S_{\mathrm{im}}$ is the size of the image), the reconstruction is limited to scales between 2 and $p-1$. Most of the reconstructed objects correspond to diffuse patches of moderate absorption or to emission minima and are not relevant for the present study. We thus only keep the objects for which the contrast (ratio of the intensity deficit to the background intensity), $C$, reaches at least $15 \%$ at one position of the object. The resulting lists at 7 and $15 \mu \mathrm{m}$ are then cross-identified on the basis of spatial coïncidence.

Figure 5 shows the result of the emission clipping in the 9.85-0.20 field at $7 \mu \mathrm{m}$. Diffuse and more compact structures, partly connected, are present. The reconstructed

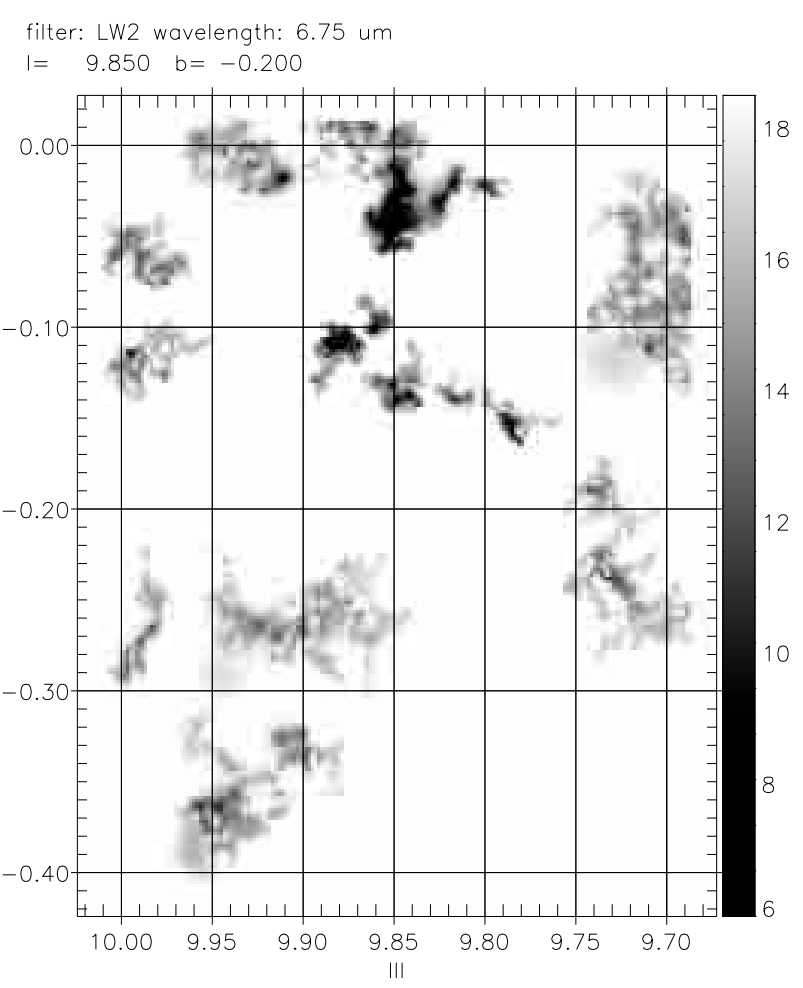

Fig. 6. Reconstructed LW2 image of the 9.85-0.20 field using MVM

image obtained after performing all steps of MVM and applying the minimal contrast criterion, is displayed in Fig. 6. All significant structures, mostly in the upper part of the map (above $b=-0.15^{\circ}$ ), have been properly reconstructed. The isolated diffuse structures have been eliminated by the minimal contrast criterion whereas the diffuse parts spatially connected to the denser ones remain. Small structures at scale 1 , like the feature located at $l=9.92^{\circ}$ and $b=-0.08^{\circ}$, have not been reconstructed either. One of the limitations of MVM is that, using square wavelets, narrow filaments are detected as lines of scale 1 elements, and thus fall off the catalogue.

The clipped and reconstructed LW3 images (Fig. 2) are displayed in Figs. 7 and 8. Similar results to LW2 are obtained for the denser parts (but for the narrowest features) whereas differences for the diffuse regions are observed (due to the minimal contrast criterion). Contrarily to what happened with the LW2 image, the narrow structure at $l=9.92^{\circ}$ and $b=-0.08^{\circ}$ is connected with a diffuse feature; the scale of the object being then greater than 1 , it has been retained. For similar reasons, the structure at $l=10^{\circ}$ and $b=-0.28^{\circ}$ that was detected in the LW2 image, has been dropped in the LW3 image.

Apart from these boarderline cases, which illustrate the completeness limit of our catalogue, but certainly could be repaired in a future, more refined analysis, it is satisfying to remark that all large and most contrasted features, which represent the target of the present study, are similarly extracted in both images. Another limitation of our "local" detection is that dark objects larger than the size of the ISOGAL images $\left(\simeq 10-30^{\prime}\right)$ will escape 


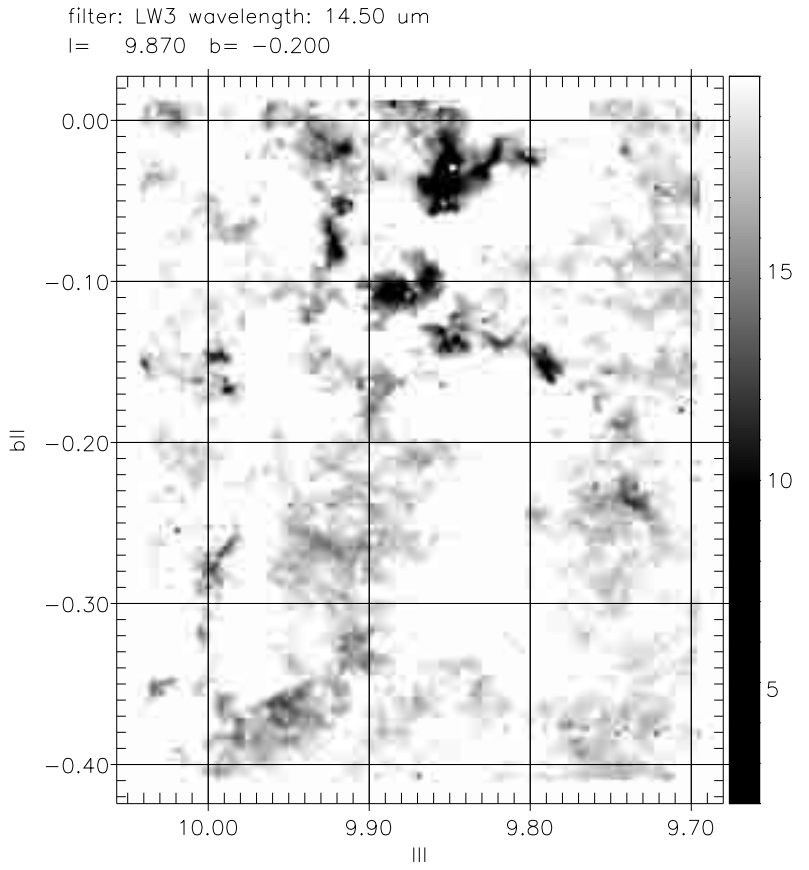

Fig. 7. $14 \mu \mathrm{m}$ LW3 image of the 9.85-0.20 field after clipping of all positive structures

detection. The risk also exists that a background area in the neighbourhood of a bright emissive region may be mistaken for a dark object. These false detections however will pop up at a later stage of the analysis. It should be kept in mind that a study based on sky darkness is biased in favour of the most "visible" objects, namely the nearest.

We applied the procedure just described to all ISOGAL images. The number of dark objects found by this procedure for the LW2 and LW3 filters is about 400, whereas it is about 300 for LW9, 200 for LW6 and 200 for LW5. The final number of cross-identified objects for each of the three subsets is around 250 for LW2-LW3, around 100 for LW6-LW9, and around 100 for LW5-LW9. In order to build on sufficiently stable grounds, we only consider the cross-identified objects in the analysis below.

\subsection{Morphology}

The clouds have a diverse multiscale morphology, that we characterise with simple indicators: surface, aspect ratio, filling factors and shape indicator, computed on masks obtained by thresholding the reconstructed objects below 3 different values: 1,3 and $5 \mathrm{MJy} \mathrm{sr}{ }^{-1}$ at $15 \mu \mathrm{m}$. By choosing the reconstructed objects we lose some details, but this also smoothes out part of the noise.

The aspect ratio is defined as:

$A_{\mathrm{r}}=\sqrt{J_{\max } / J_{\min }}$

where $J_{\max }$ is the largest principal moment of inertia and $J_{\min }$ the smallest. Aspect ratios around 1 are representative of roughly spherical objects while larger ratios characterise elongated shapes. To obtain the moments of

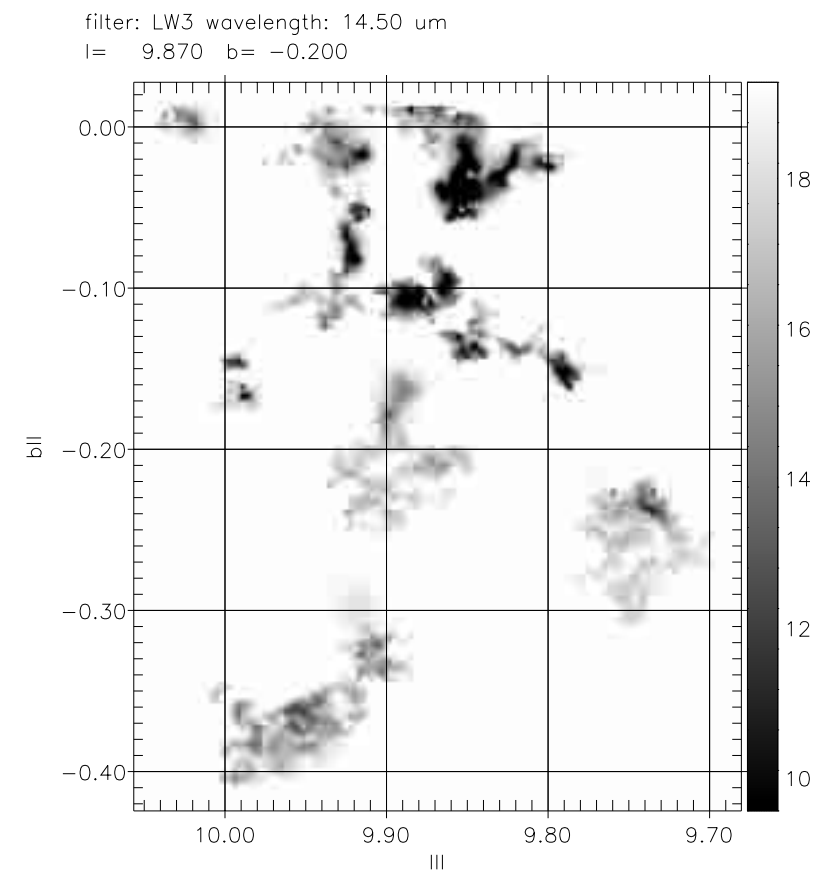

Fig. 8. Reconstructed LW3 image of the 9.85-0.20 field

inertia, we calculate the inertia centre of the object, then the three moments:

$\int x^{2} \mathrm{~d} I, \int y^{2} \mathrm{~d} I, \int x y \mathrm{~d} I$

where $I$ is the intensity, $x$ and $y$ the coordinates. These three moments define the symmetric matrix of inertia whose two eigenvalues are the two principal moments of inertia.

The filling factor is defined by:

$F_{\mathrm{f}}=\frac{S}{\pi \sqrt{J_{\max } J_{\min }}}$.

It is equal to 1 for a disk and aims at characterising how the objects fill the area over which they extend.

As shape indicator, we use the parameter $S_{\mathrm{i}}$ :

$S_{\mathrm{i}}=\frac{4 \sqrt{S}}{P}$

where $P$ is the perimeter. It gives an estimate of the relative contour weight. As a matter of comparison, $S_{\mathrm{i}}$ is equal to 1 for a square, $2 \sqrt{n} /(n+1)$ for a rectangle of length $n$ and width $1,1 / \sqrt{n}$ for $n$ disconnected squares.

The histograms of the 4 parameters $S, A_{\mathrm{r}}, F_{\mathrm{f}}$ and $S_{\mathrm{i}}$ are displayed in Figs. 9 and 10, for the 3 threshold values.

The cloud surfaces, $S$, vary from less than 1 to over 30 square arcmin and depend strongly on the threshold. For the lowest threshold value, the typical surface is about 5 to 20 square arcmin whereas it is barely 1 square arcmin for the largest threshold. This shows that the clouds are not uniform but present small denser parts and broad diffuse parts. 

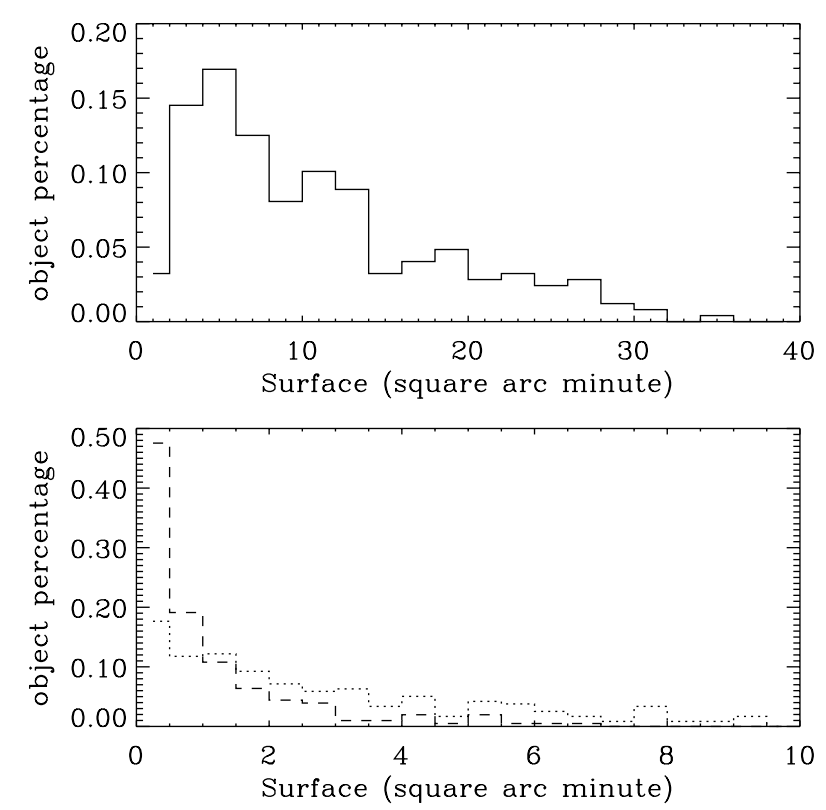

Fig. 9. Clouds surface. Full line: $1 \mathrm{MJy} \mathrm{sr}^{-1}$ threshold. Dotted line: $3 \mathrm{MJy} \mathrm{sr}^{-1}$ threshold. Dashed line: $5 \mathrm{MJy} \mathrm{sr}^{-1}$ threshold
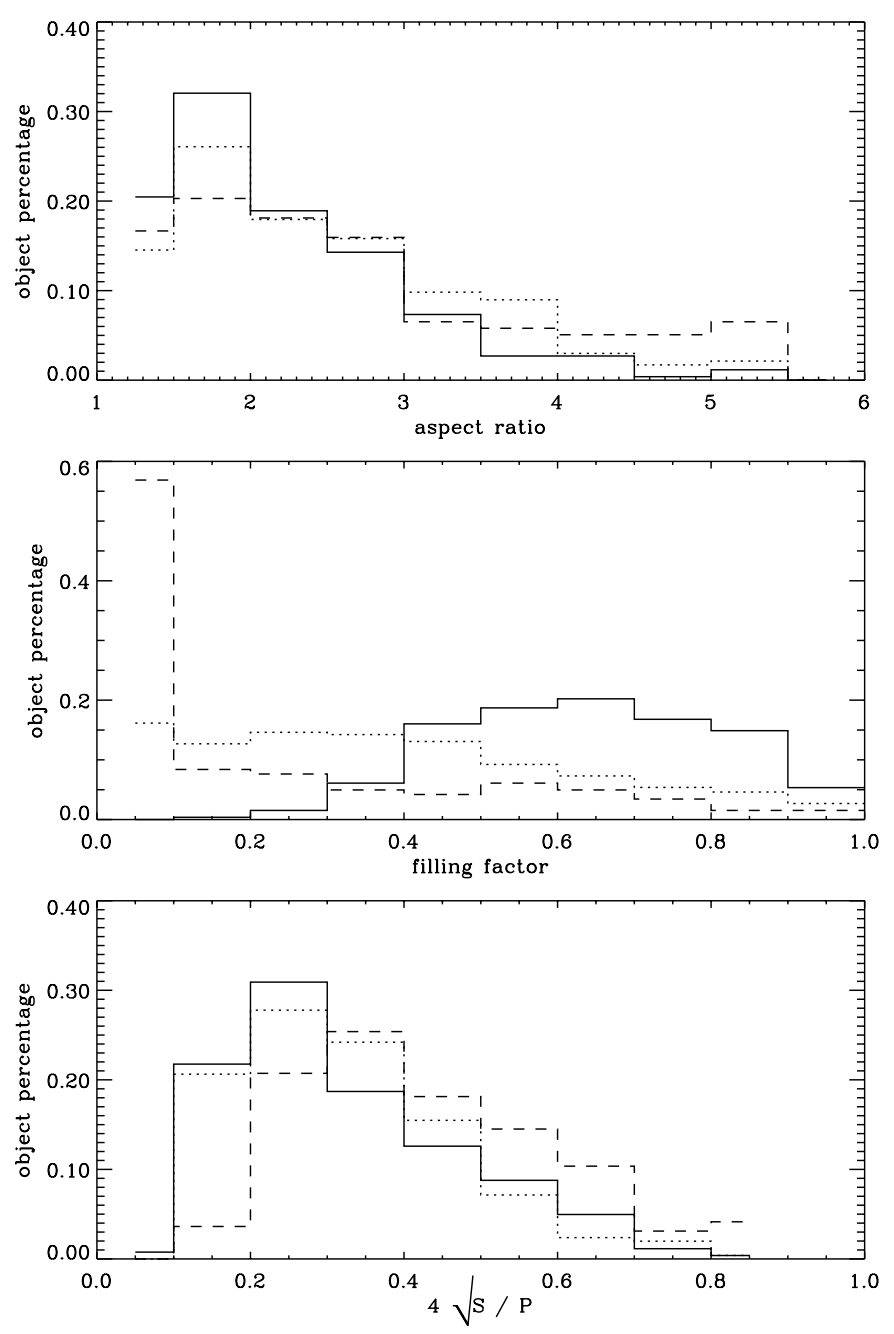

Fig. 10. Aspect ratio, filling factor and shape indicator of the clouds, for three different thresholds (see Fig. 9)
The aspect ratio ranges from 1 to 6 and the peak of the distribution is around 1.5-2. The aspect ratio increases with the threshold value. The condensed structures are thus mostly elongated. This does not exclude sheets, which are more easily detected in absorption when seen edge on.

The filling factor depends a lot on the threshold value. Its typical value is about $0.5,0.2$ and 0.1 respectively for the three thresholds considered. The denser parts do not fill the surface, meaning that the dark structures contain more than one single dense part. Therefore the dark objects present an internal structure composed by a broad envelope with few denser parts inside.

The typical value of $S_{\mathrm{i}}$ is 0.2 , meaning that the objects are very fragmented with a lot of contours. 25 isolated squares or a rectangle of aspect ratio 100 would be necessary to get the same value of $S_{\mathrm{i}}$. Its value increases somewhat when larger thresholds are considered, which is consistent with the picture of few small dense cores in a more diffuse envelope. As shown in Teyssier et al. (2001), these objects indeed turn out to be the most condensed cores of giant molecular clouds located between the Sun and the galactic "tangent point" (position where the line of sight is tangent to a galactic circle, roughly mid way through the disk). We shall now see that many of them indeed lie quite deep in the disk.

\subsection{I, b distribution of the mid-IR dark-clouds}

The $(l, b)$ distribution of the dark objects detected in LW3 and LW9 is displayed in Fig. 11. The dark object number density roughly follows the distribution of the average background intensity. This correlation may be favoured by an observational bias: the background becomes very low at large longitude, making absorbing clouds harder to detect, if they are present. The sensitivity is high enough however for this bias not to be effective in regions above $10 \mathrm{MJy} \mathrm{sr}^{-1}$ at $15 \mu \mathrm{m}$. Confusion on the contrary might prevent some detections in the most populated (brightest) areas. The number density of these condensed objects seems anyway to follow the galactic distribution of young population components as traced e.g. in CO surveys: peak in the centre and an excess around $\pm 30^{\circ}$ corresponding to the longest lines of sight through the molecular ring (Solomon et al. 1979; Cohen et al. 1980; Sanders et al. 1984). The apparent rough proportionality between the dark clouds number density and the average mid-IR background intensity, although not astounding, cannot be straightforwardly interpreted.

Follow-up studies based on molecular spectroscopy (Teyssier et al. 2001) and near infrared IJK-band star counts agree on distance evaluations in the range $2-8 \mathrm{kpc}$. A significant fraction of the dark cores detected belongs to the molecular ring or to innermore regions, in agreement with the conclusion of the MSX galactic survey (Egan et al. 1998).

\section{Dark clouds in the inner Galaxy (LW2-LW3)}

This section is dedicated to a statistical study of the 7 and $15 \mu \mathrm{m}$ brightness fluctuations within the 

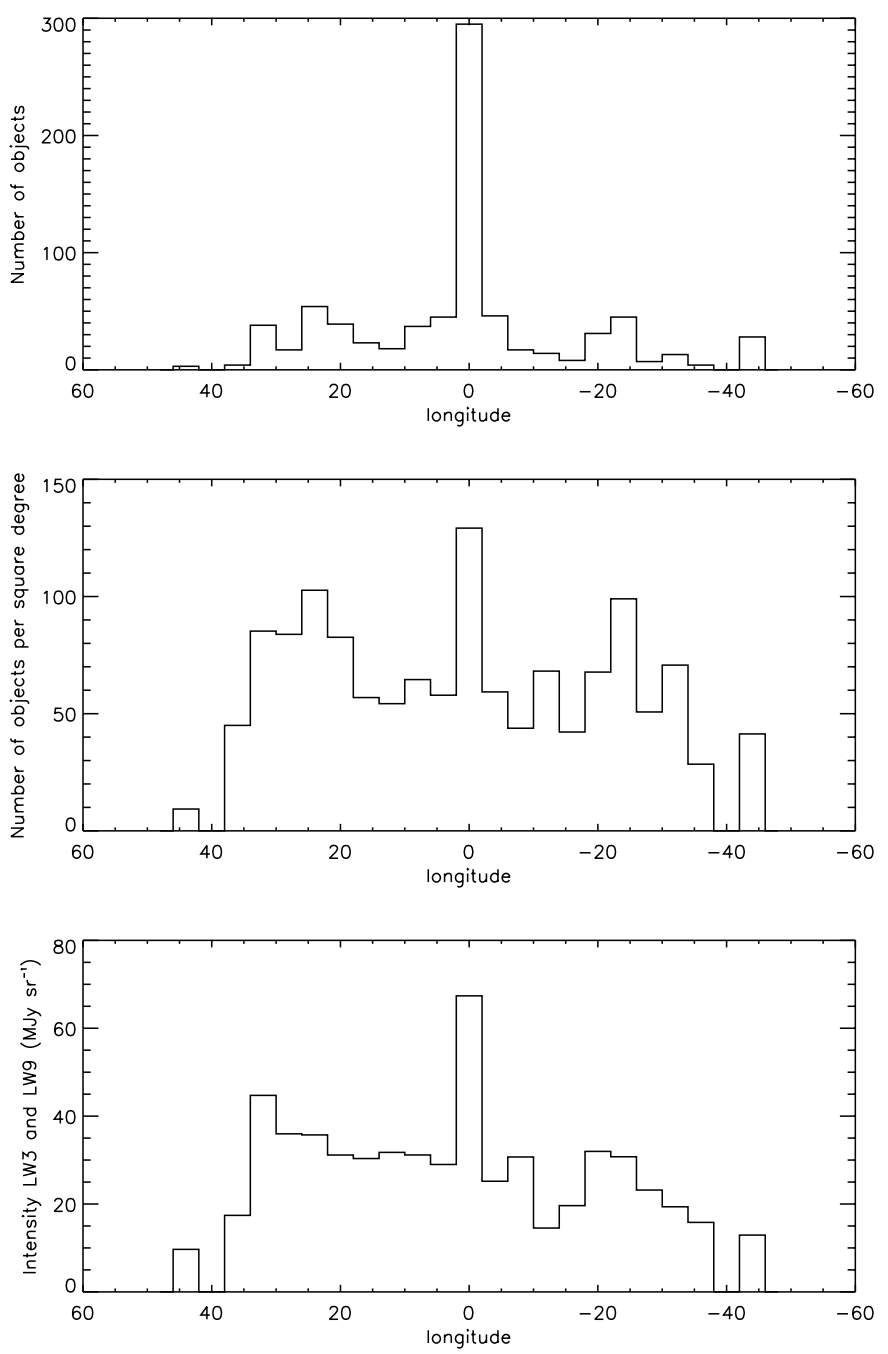

Fig. 11. Distribution of the dark objects seen in LW3 and in LW9 with galactic longitude for $|b|<0.4^{\circ}$. First top histogram: no normalisation is applied. Second histogram: normalisation by the observed surface. For comparison the average background intensity in LW3 and LW9 filters has been displayed in the lower panel

boundaries of each of the dense cores of the LW2-LW3 catalogue. The Galactic Centre samples will be considered separately (Sect. 6). Average estimates of the opacity ratio $\alpha=\tau_{\mathrm{LW} 2} / \tau_{\mathrm{LW} 3}$ are derived from the correlation of these brightness fluctuations. Absolute opacities will be derived for a few clouds in Sect. 5 .

\subsection{Intensity fluctuations}

After an accurate image registering using the ISOGAL star catalogues, scatter diagrams of 7 and $15 \mu \mathrm{m}$ intensities are plotted for all objects. The examples given in Fig. 12 show that the dark pixels (upper panel) significantly depart from the average intensity correlation represented by the straight line. The second plot (bottom panel) indeed includes a number of pixels surrounding the dark object. These external pixels follow the average correlation ( 7 to $15 \mu \mathrm{m}$ ratio $\simeq 1.2$, see Fig. 3 ).
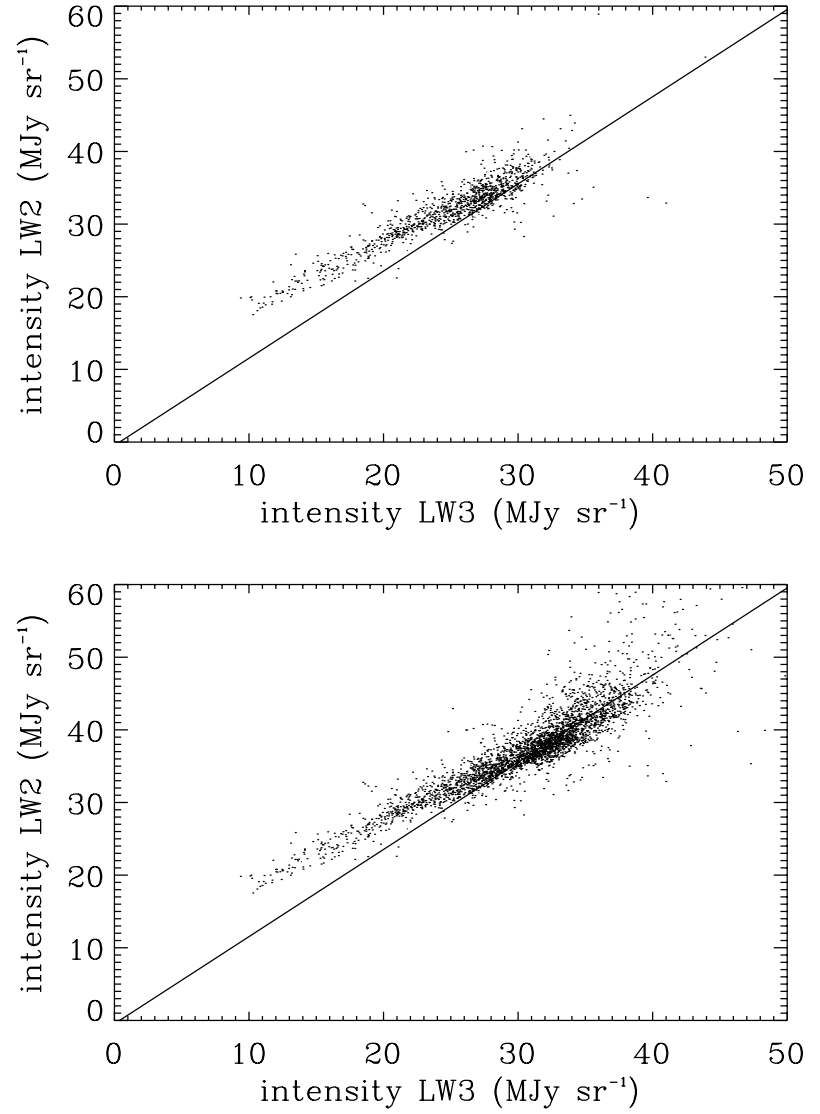

Fig. 12. Scatter diagram of LW2 versus LW3 intensity for the dark feature $\mathrm{DF}+9.85-0.05$. In the upper panel pixels within the mask given by the wavelet extraction (threshold equal to $2 \mathrm{MJy} \mathrm{sr}^{-1}$ ) have been used whereas the bottom panel includes all pixels in a larger rectangular area encompassing the object. The straight line represents the average colour $($ ratio $\simeq 1.2)$

The departure of the dark pixels from the average correlation is interpreted as inverse reddening ("blue-ing") due to differential absorption in the 2 wavelength bands. The magnitude of the effect implies that the attenuation in both bands is different and significant.

We assume in the following that the pixel intensity fluctuations, within the boundaries of a given object $I_{i}$ ( $i=1$ for the $15 \mu \mathrm{m}$ filter, $i=2$ for the $7 \mu \mathrm{m}$ filter) are dominated by fluctuations of the absorption within the object, and so define a non absorbed intensity $I_{\mathrm{T} i}$ that will be estimated with a median filter in a $12^{\prime} \times 6^{\prime}$ strip around the object. This strip is extended if needed, in order to include a minimum of 5000 pixels not belonging to the cloud. The definition of the pixel dependent contrast follows, for each filter:

$C_{i}=\frac{I_{\mathrm{T} i}-I_{i}}{I_{\mathrm{T} i}}$

In order to quantitatively interpret the 7 to $15 \mu \mathrm{m}$ contrast correlation in the dark objects, we simply model the pixel intensity as superposition of emission from behind the cloud, $I_{\mathrm{b}, i}$, cloud opacity $\tau_{i}$ and emission from material in front of the cloud, $I_{\mathrm{f}, i}$. Additional absorption, 
behind or in front of the cloud is ignored at this stage. The pixel intensity then simply writes:

$I_{i}=I_{\mathrm{b} i} \exp \left(-\tau_{i}\right)+I_{\mathrm{f} i}$.

At the basic interpretation level suggested, we assume that $I_{\mathrm{b}, i}$ and $I_{\mathrm{f}, i}$ are uniform over the cloud, the intensity fluctuations being attributed to fluctuations of $\tau_{i}$. Under these assumptions $I_{\mathrm{T} i}=I_{\mathrm{b} i}+I_{\mathrm{f} i}$ and the "darkness" contrast writes:

$C_{i}=\beta_{i}\left(1-\exp \left(-\tau_{i}\right)\right)$,

where $\beta_{i}=I_{\mathrm{b} i} / I_{\mathrm{T} i}$, is at most equal to 1 . The contrast $C_{i}$ always satisfies $\mathrm{C}_{i}<\tau_{i}$. The uniformity of the diffuse emission colour shown by Figs. 3 and 4 favours the additional assumption $\beta_{1}=\beta_{2}$. These simple relations are now used to constrain the values of $\tau_{i}$ for the LW2-LW3 sample. Part of the assumptions will be relaxed in later sections.

\subsection{Determination of the 7 to $15 \mu \mathrm{m}$ opacity ratio}

\subsubsection{7 to $15 \mu \mathrm{m}$ contrast ratio}

The 7 to $15 \mu \mathrm{m}$ darkness contrast ratio writes, in the simple workframe just introduced:

$\frac{C_{2}}{C_{1}}=\frac{\beta_{2}}{\beta_{1}} \frac{1-\exp \left(-\tau_{2}\right)}{1-\exp \left(-\tau_{1}\right)} \simeq \frac{1-\exp \left(-\tau_{2}\right)}{1-\exp \left(-\tau_{1}\right)}$.

We define:

$f\left(\alpha, \tau_{1}\right)=\frac{1-\exp \left(-\alpha \tau_{1}\right)}{1-\exp \left(-\tau_{1}\right)}$.

Let us note that if $\alpha \leq 1, \alpha \leq f(\alpha, \tau) \leq 1$, if $\alpha \geq 1$, $1 \leq f(\alpha, \tau) \leq \alpha$.

In order to boost the signal to noise ratio, the calculation of the contrast ratio is achieved using larger pseudopixels. Pseudo pixels are averages of 10 pixels, grouped in order of decreasing contrast at $15 \mu \mathrm{m}$. The resulting values are noted $\left\langle C_{i}\right\rangle(i=1,2)$. The distribution of these values and of their ratio are shown in Fig. 13: restricted to the darkest pseudo-pixel of each object (left panels) or including up to 40 pseudo-pixels per object (right panels).

The distributions of $\left\langle C_{1}\right\rangle$ and $\left\langle C_{2}\right\rangle$ peak respectively around 0.3 and 0.25 . As $\left\langle C_{i}\right\rangle$ is smaller than the opacity $\tau_{i}$, we can infer that the maximum opacity for most of the dark objects is greater than 0.3 at $14 \mu \mathrm{m}$ and greater than 0.2 at $7 \mu \mathrm{m}$. Some of them have values of $\left\langle C_{1}\right\rangle$ and $\left\langle C_{2}>\right.$ larger than 0.5 indicating probably more massive and/or closer objects. A few examples are studied in the next section. The histograms of the 7 to $15 \mu \mathrm{m}$ contrast ratio relatively sharply peak around $0.75 \pm 0.15$ implying an opacity ratio $\alpha$ smaller than $\simeq 0.8$. Let us also remark that the full width at half maximum of the $\left\langle C_{1}\right\rangle$ histograms is about 0.15 whereas it is about 0.1 for $\left\langle C_{2}\right\rangle$. The ratio of the two is expected to be at first order roughly proportional to $\alpha$, thus consistent with an upper limit around 0.75 .
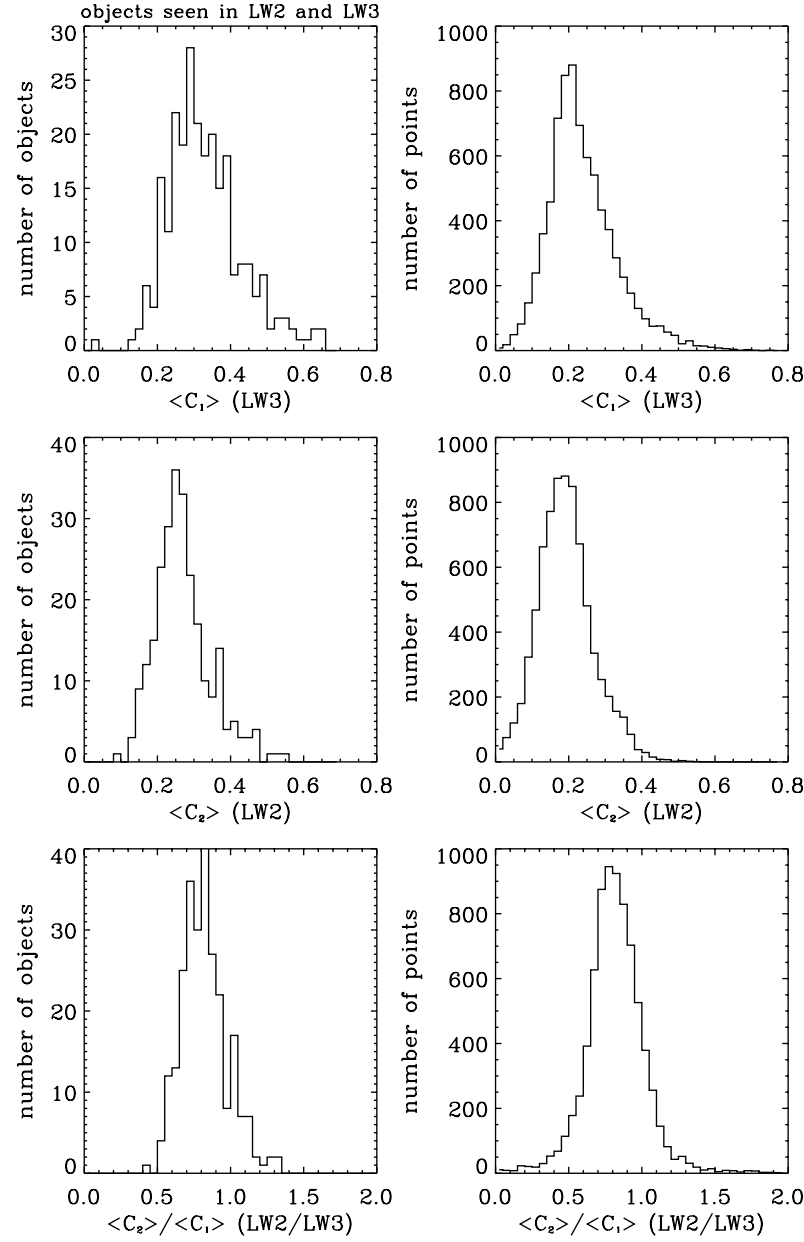

Fig. 13. Histograms of $\left\langle C_{1}\right\rangle$ (LW3), $\left\langle C_{2}\right\rangle$ (LW2) and $\left\langle C_{2}\right\rangle /\left\langle C_{1}\right\rangle$. Left panels: darkest values obtained in each cloud (see text). Right panels: all values obtained (limited to 40) in each cloud

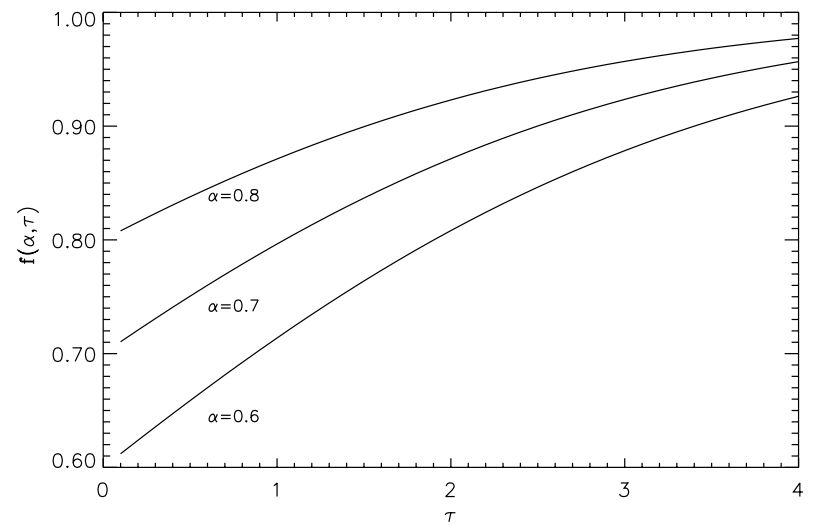

Fig. 14. $f$ plotted as a function of $\tau$ for three values of $\alpha$

\subsubsection{7 to $15 \mu \mathrm{m}$ opacity ratio}

A large fraction of dark lines of sight have contrast ratios $<C_{2}>/<C_{1}>\simeq 0.75 \pm 0.15$ and $15 \mu \mathrm{m}$ opacities $\tau \geq 0.3$. Assuming that the actual $\tau_{1}$ values are in the range $[0.5,1]$, a prediction of $\alpha$ can be made from Eq. (5), under the assumption $\beta_{2} / \beta_{1} \simeq 1$, namely $\alpha=0.7 \pm 0.15$ as shown in Fig. 14. This prediction is in agreement with the 

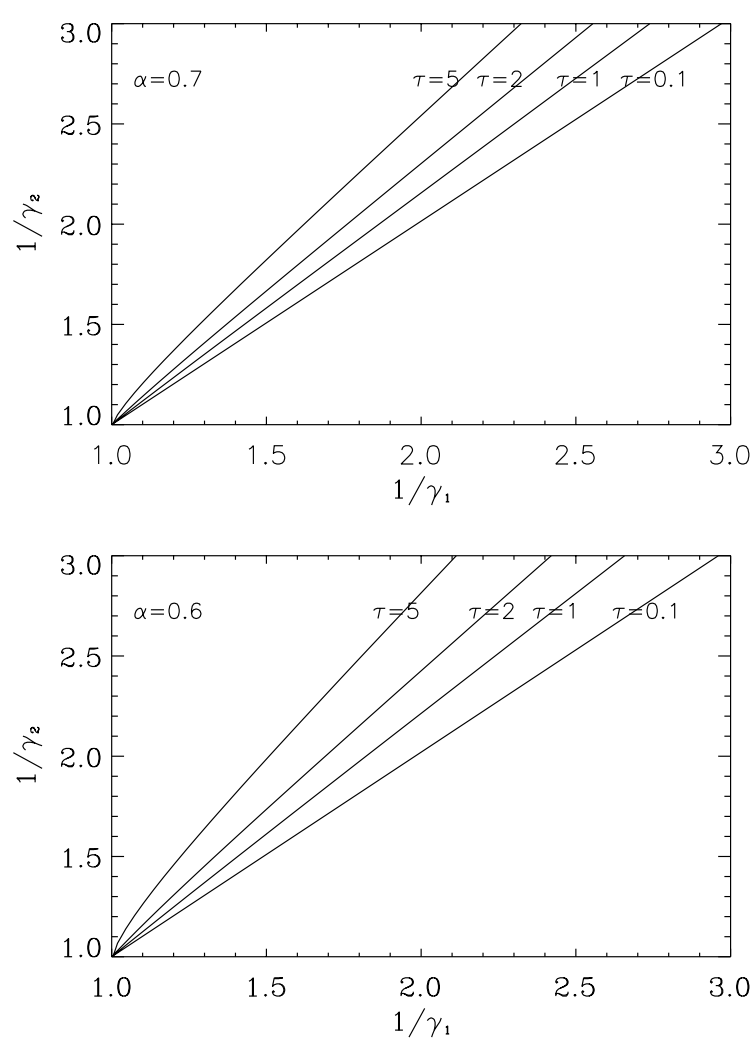

Fig. 15. $1 / \gamma_{2}$ vs. $1 / \gamma_{1}$ for $\alpha$ equal to 0.6 and 0.7 and $\tau_{1, \mathrm{~m}}$ equal to $0.1,1,2,5$

extinction curve derived by Draine \& Lee (1984), for which a value $\alpha \simeq 0.7$ is expected. A similar conclusion was reached by Abergel et al. (1998) for a dense core in the solar neighbourhood. Contrarily, it is less consistent with the observational results of Lutz (1998) who used hydrogen recombination lines to constrain the extinction law at midinfrared wavelengths towards the Galactic center. His results lead to a larger opacity ratio $\alpha \simeq 0.9-1.1$. It would be interesting to better constrain our estimate of $\alpha$. This will be attempted on individual objects, refining on the non-linear dependence of $\left\langle C_{2}\right\rangle$ with $\left\langle C_{1}\right\rangle$.

Before turning to these objects, let us point out that $10 \%$ of the data points have contrast ratios smaller than 0.6 , and $10 \%$ larger than 1 , hence significantly different from the median value 0.8 . Several explanations can be put forward:

- uncertainty on the values of $I_{\mathrm{T} 1}$ and $I_{\mathrm{T} 2}$;

- detection of false dark objects in fluctuating emission areas (HII region...);

- gradient of the colour ratio $\left(\beta_{1} / \beta_{2} \neq 1\right)$ due for example to diffuse extinction on the line of sight (see Sect. 6.3) or to local emission with different colour;

- variation of $\alpha$ itself.

It is likely that these four situations somewhat contribute to the discrepant measurements.
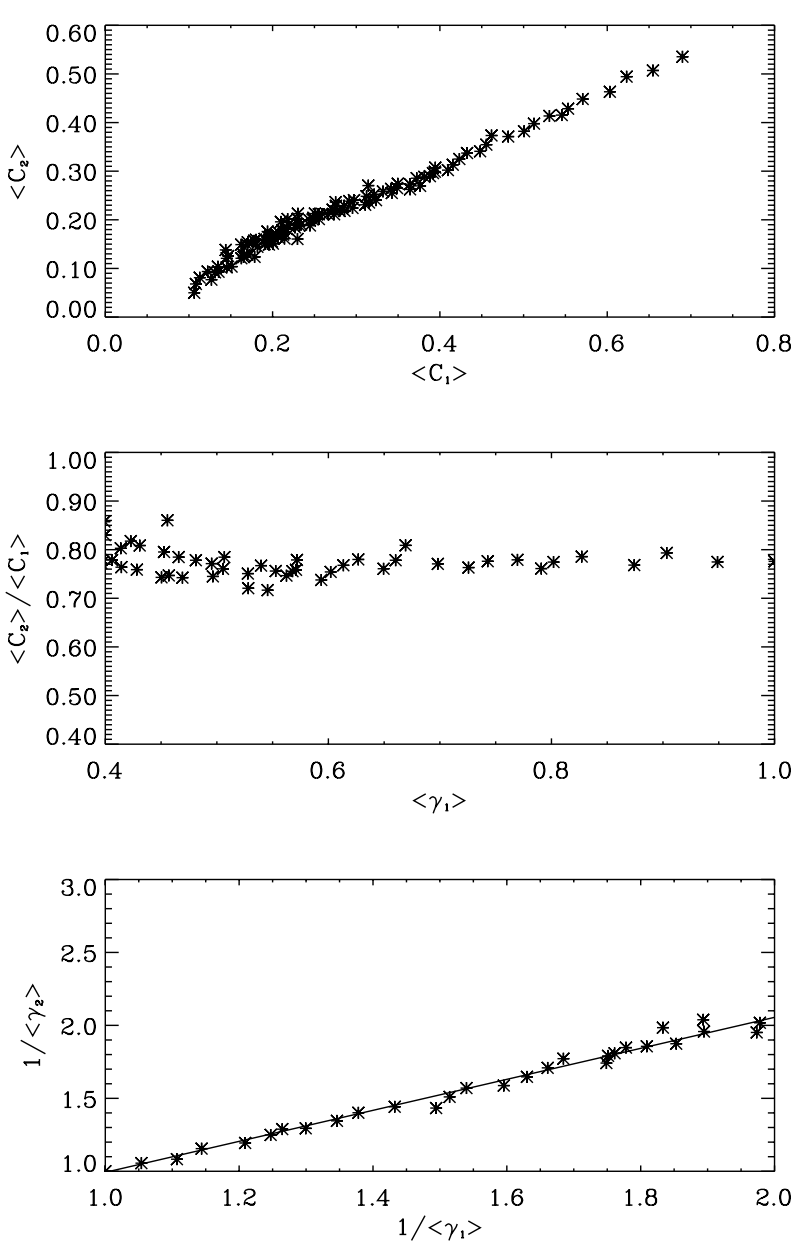

Fig. 16. Plot of $\left\langle C_{2}\right\rangle$ vs. $\left.\left\langle C_{1}\right\rangle,\left\langle C_{2}\right\rangle /<C_{1}\right\rangle$ vs. $<\gamma_{1}>, 1 /<\gamma_{2}>$ vs. $1 /<\gamma_{1}>$ for $\mathrm{DF}+09.85-0.05$

\section{Special cases}

Instead of looking at average contrast ratios, which yield accurate trends, but smooth out the opacity corrections, we now turn to the spatial contrast modulation within some of the darkest objects.

\subsection{Method}

According to the simple interpretation frame of the contrast fluctuations proposed in Sect. $4, C_{i}$ raises from 0 to $\beta_{i}$ and the contrast ratio $C_{2} / C_{1}$ from $\alpha \beta_{2} / \beta_{1}$ to $\beta_{2} / \beta_{1}$, with increasing opacities $\tau_{i}$. It follows that the maximum relative variation of the contrast ratio gives an upper limit to $\alpha$ irrespective of additional assumptions on $\beta_{2} / \beta_{1}$. Conversely, if $\alpha$ is assumed to be known, the maximum relative variation of the contrast ratios yields an estimate of the maximum opacity through the cloud.

We define a normalised contrast, equal to 1 at the darkest position:

$\gamma_{i}(\tau)=\frac{C_{i}(\tau)}{C_{i, \max }}=\frac{1-\exp \left(-\tau_{i}\right)}{1-\exp \left(-\tau_{i, \max }\right)}$.

The relative variation of $\gamma_{1}$ and $\gamma_{2}$ is shown in Fig. 15 for different values of $\tau_{i, \max }$, and for $\alpha=0.6$ and $\alpha=0.7$. 
Table 1. Maximum opacity estimate inside six objects for two values of $\alpha$

\begin{tabular}{|c|c|c|c|c|c|}
\hline Coordinates $(l, b)$ & $C_{1, \max }$ & $C_{2, \max }$ & $\left(1 / \gamma_{2}\right.$ vs. $\left.1 / \gamma_{1}\right)$ slope & $\alpha=0.7: \tau_{1, \max },\left(\beta_{1}, \beta_{2}\right)$ & $\alpha=0.6: \tau_{1, \max },\left(\beta_{1}, \beta_{2}\right)$ \\
\hline \hline$(4.35,-0.05)$ & 0.42 & 0.34 & $1.4 \pm 0.2$ & $3 \pm 2,(0.43,0.37)$ & $2 \pm 1,(0.45,0.42)$ \\
\hline$(9.85,-0.02)$ & 0.67 & 0.50 & $1.1 \pm 0.2$ & $1 \pm 1,(1,1)$ & $0.8 \pm 1,(1.2,1.3)$ \\
\hline$(12.42,-0.15)$ & 0.52 & 0.36 & $1.2 \pm 0.2$ & $1.5 \pm 1.5,(0.67,0.55)$ & $1 \pm 1,(0.82,0.71)$ \\
\hline$(15.06,0.08)$ & 0.67 & 0.52 & $1.35 \pm 0.2$ & $2.5 \pm 1.5,(0.73,0.63)$ & $1.5 \pm 1,(0.86,0.87)$ \\
\hline$(18.55,-0.15)$ & 0.47 & 0.3 & $1.45 \pm 0.2$ & $3.5 \pm 2,(0.54,0.4)$ & $2.5 \pm 1,(0.51,0.38)$ \\
\hline$(30.01,-0.40)$ & 0.36 & 0.26 & $1.35 \pm 0.2$ & $2.5 \pm 1.5,(0.39,0.31)$ & $1.5 \pm 1,(0.46,0.44)$ \\
\hline
\end{tabular}
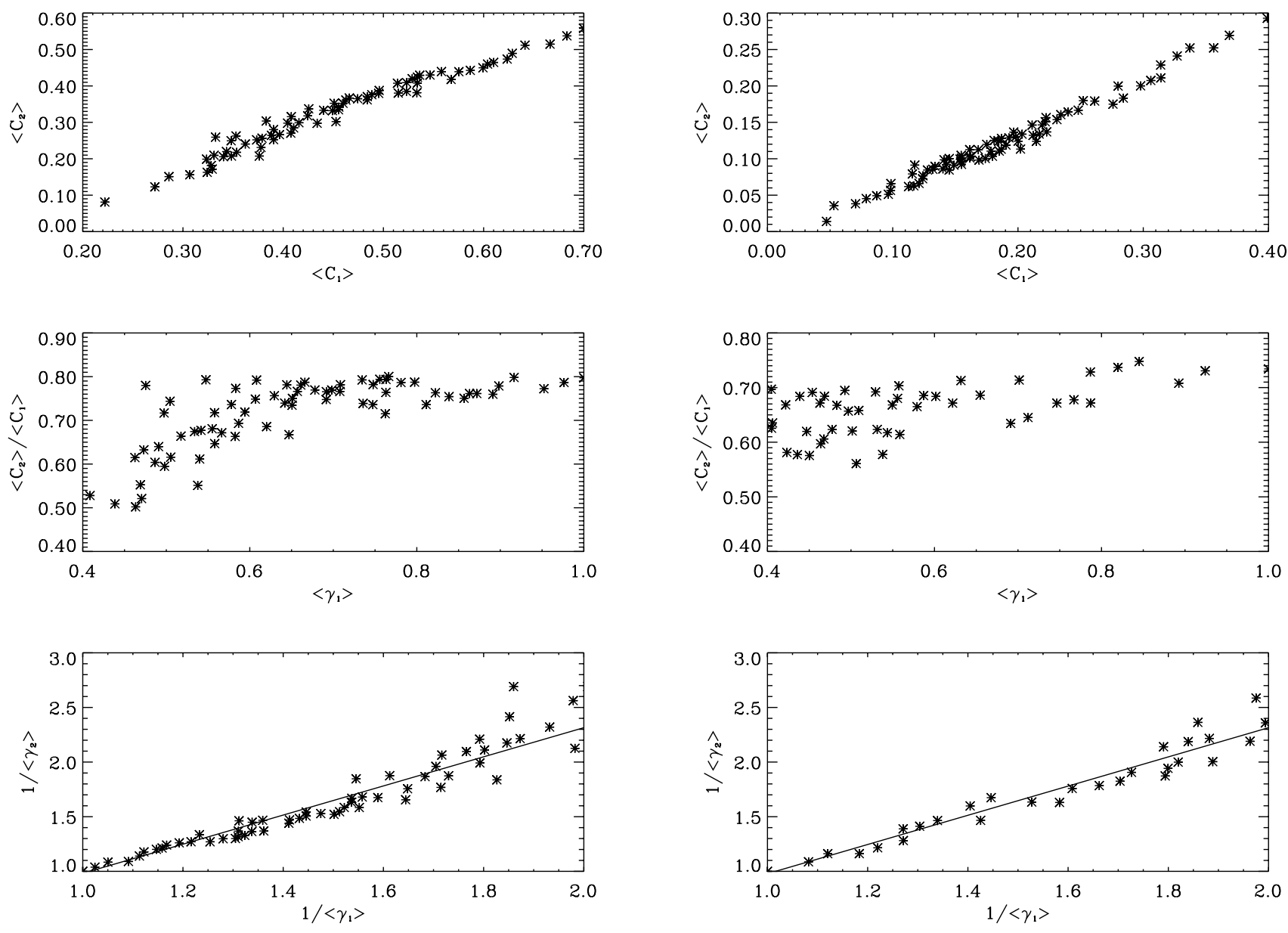

Fig. 17. Same as Fig. 16 for $\mathrm{DF}+15.05+0.08$

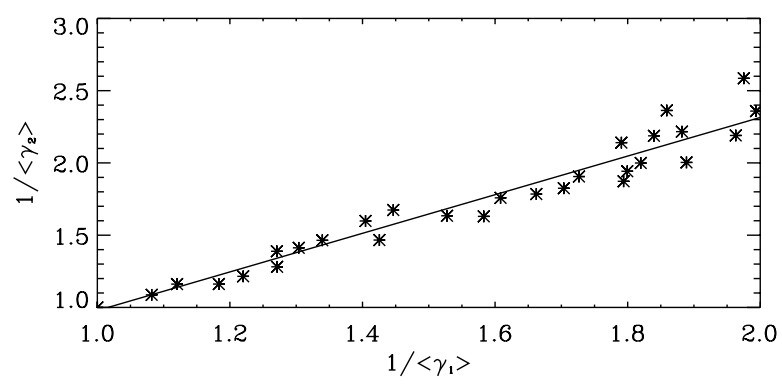

Fig. 18. Same as Fig. $16 \mathrm{DF}+30.00-0.39$

The sensitivity on $\tau_{i, \max }$ is more obvious when using inverse contrasts, adopted for these plots. These simple behaviours are now used for examining 6 examples of relatively good signal to noise ratio.

\subsection{Results}

Contrasts estimated in the pseudo-pixels defined in Sect. 4.2 are used in three different diagrams: $\left\langle C_{1}\right\rangle$ vs. $<C_{2}>$ scatter diagram, $\left\langle C_{2}\right\rangle /<C_{1}>$ as a function of $\left\langle\gamma_{1}\right\rangle$, and $1 /<\gamma_{1}>$ vs. $1 /<\gamma_{2}>$ scatter diagram.

In the three cases presented in Figs. $16-18$, as predicted by the model (Fig. 14) the ratio $\left\langle C_{2}\right\rangle /\left\langle C_{1}\right\rangle$ increases slightly with $\tau$. For DF+09.85-0.05 (Fig. 16) the

value of $\left\langle C_{2}>/<C_{1}>\right.$ does not significantly increase. Its value is $\simeq 0.78$ suggesting $\alpha \leq 0.78$ and $\tau_{1} \leq 1$. For $\mathrm{DF}+15.05+0.08$ (Fig. 17), $\left\langle C_{2}\right\rangle /\left\langle C_{1}\right\rangle$ varies between less than 0.65 and 0.8 suggesting a value of $\alpha \leq 0.7$ and a darker object with $\tau_{1} \geq 1$. For $\mathrm{DF}+30.01-0.40$ (Fig. 18) $<C_{2}>/<C_{1}>$ ranges from 0.65 to less than 0.75 . This is compatible with a value of $\alpha \leq 0.7$ and a value of $\tau_{1}$ larger than 1 .

These three special cases are in good agreement with a value $\alpha=0.7 \pm 0.1$, close to Draine \& Lee's prediction (1984). Most probably, the variations are due to values of $\beta_{2} / \beta_{1} \neq 1$.

Best estimates of $\tau_{1, \max }, \beta_{1}$ and $\beta_{2}$ are given in Table 1 for the 6 examples retained, assuming either $\alpha=0.7$ 

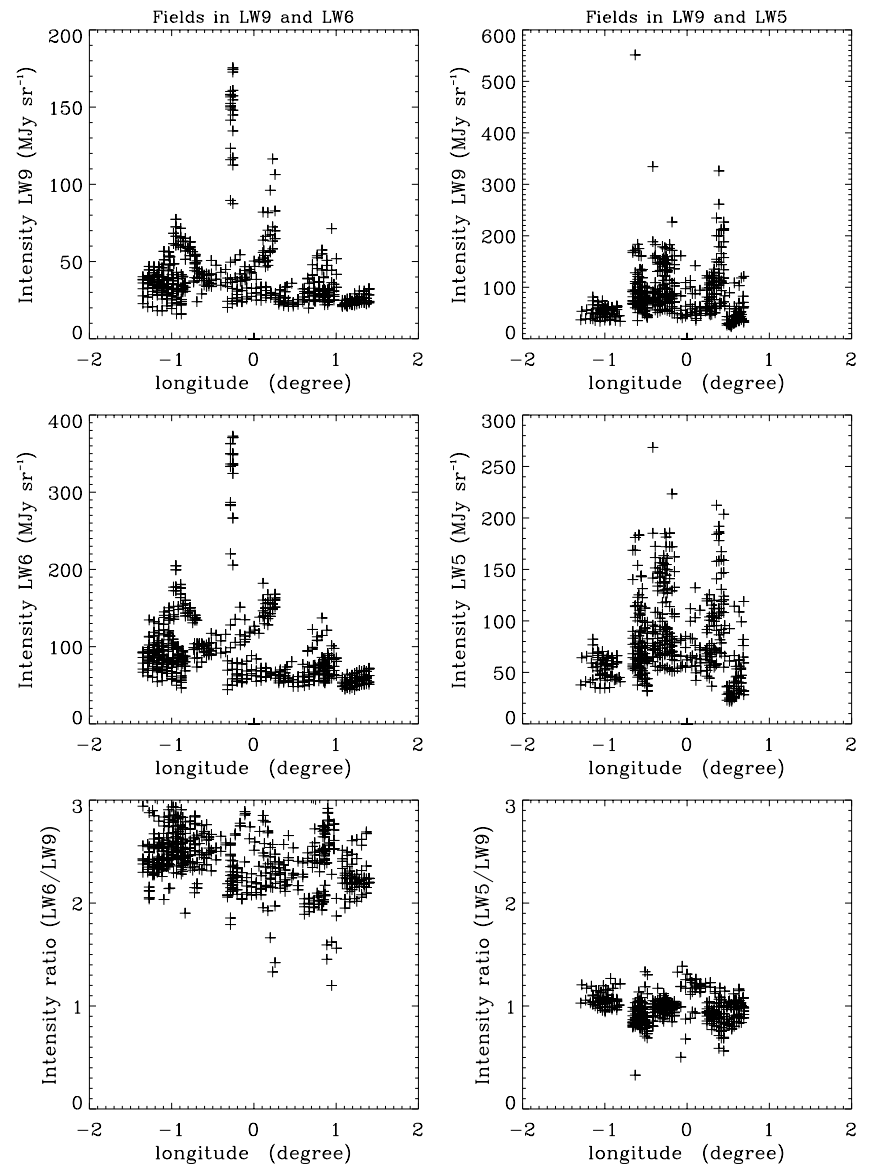

Fig. 19. Intensity (median value in a regions of $35 \times 35$ pixels) of the fields seen in LW9-LW6 (left panels) and LW9-LW5 (right panels) as a function of the Galactic longitude

or $\alpha=0.6$. The accuracy of the slope estimates in the $1 / \gamma_{2}$ vs. $1 / \gamma_{1}$ diagrams is limited by the accuracy of the background estimate. We use different methods to measure its value and evaluate the accuracy of the slope estimates to be $\simeq \pm 0.2$.

The maximum $15 \mu \mathrm{m}$ optical depths range from 1 to 3.5 (or maybe more, but the methods lack sensitivity to larger values) for $\alpha=0.7$ and from less than 1 to 2.5 for $\alpha=0.6$. The values of $\beta_{1}$ and $\beta_{2}$ range from $\simeq 0.4$ for $\mathrm{DF}+30.01-0.40$ to $\simeq 1$ for $\mathrm{DF}+09.85-0.02$. This suggests that $\mathrm{DF}+09.85-0.02$ is the nearest cloud whereas $\mathrm{DF}+30.01-0.40$ should be the most distant one. The $\beta_{2} / \beta_{1}$ ratio is consistently around 0.8 if $\alpha=0.7$ and 1 if $\alpha=0.6$, but some variation is not unlikely (see Figs. 3 and 4). The opacities inferred can be used with an extinction law (Draine \& Lee 1984; Lutz et al. 1996; Lutz 1998) to derive column densities. This issue will be revisited in Teyssier et al. (2001) where correlation with radio data will also be addressed. The ratio $A_{\lambda} / A_{v}$ at $7 \mu \mathrm{m}$ from Lutz's extinction is $\simeq 4-510^{-2}$, yielding $\mathrm{H}$ column densities ranging from 0.25 to $110^{23} \mathrm{~cm}^{-2}$ for solar neighbourhood conditions. The value proposed by Draine \& Lee is $\simeq 1.5^{-2} 10^{-2}$ which leads to column densities ranging from 1 to $410^{23}$ $\mathrm{cm}^{-2}$. These values are consistent with most of the values given by Carey et al. (1998) except for the clouds
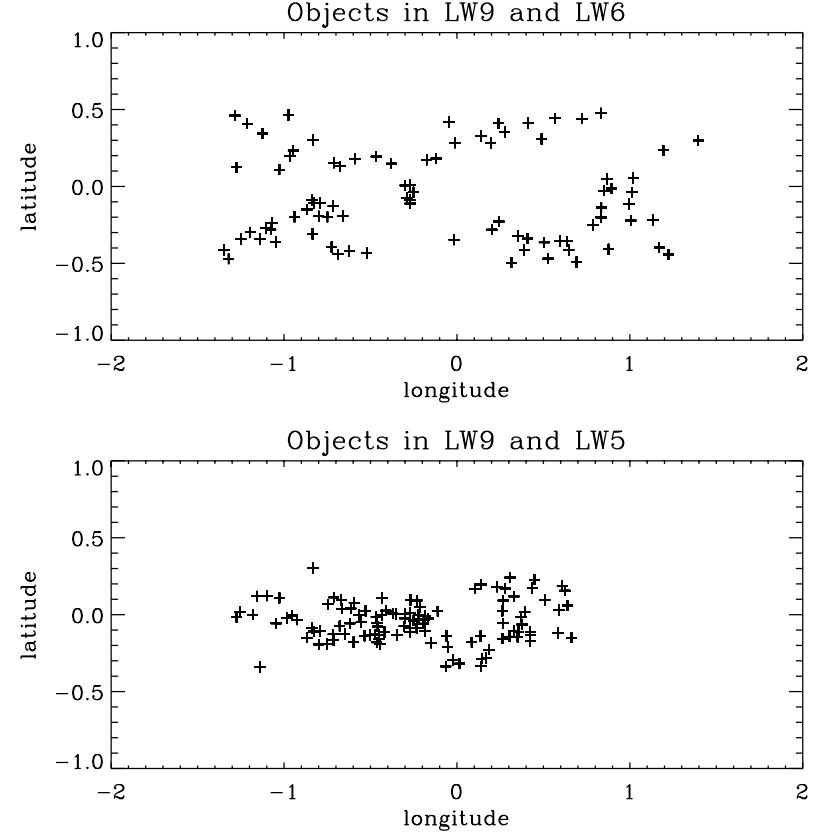

Fig. 20. Position of the clouds seen in LW9-LW6 (upper panel) and LW9-LW5 (lower panel)

located in the Galactic Centre where higher values $\left(10^{24}\right.$ $10^{25} \mathrm{~cm}^{-2}$ ) are found (see also Lis \& Menten 1998; Lis et al. 1998).

\section{Dark clouds in the Galactic Centre region}

\subsection{The observations}

In this part, we present the study of the clouds located in the Galactic Centre region observed with the narrow filters, LW5, LW6 and LW9. In the following, we call first group of observations, the observations in LW2 and LW3 presented in the previous section, the second group includes the observations in LW6 and LW9 and the third group, the observations in LW5 and LW9. Figure 19 shows the intensity (median value in square areas of $3.5 \times 3.5$ arcmin) in the fields of the second and third groups as a function of the longitude.

It ranges from 30 to $150 \mathrm{MJy} \mathrm{sr}^{-1}$ for the second group and from 50 to $200 \mathrm{MJy} \mathrm{sr}^{-1}$ for the third group. The intensity variations are more important than for the first observation group, the background is more structured. The ratio between the intensities in the two bands, LW9 and LW6, is about 2.5 whereas it is about 1 between the two bands LW9 and LW5 while it was $\simeq 1.2$ for the first observation group. This is mostly due to the relative contribution of the $7.7 \mu \mathrm{m}$ PAH feature to the emission measured in each of the $7 \mu \mathrm{m}$ filters.

The distribution in galactic coordinates of the clouds belonging to the second and third observation group are shown in Fig. 20. Clearly, the third observation group is nearer to the Galaxy Centre than the second one. The nearest clouds are located to about $0.2^{\circ}$ from the Galaxy Centre. The absorption properties of these clouds are now 

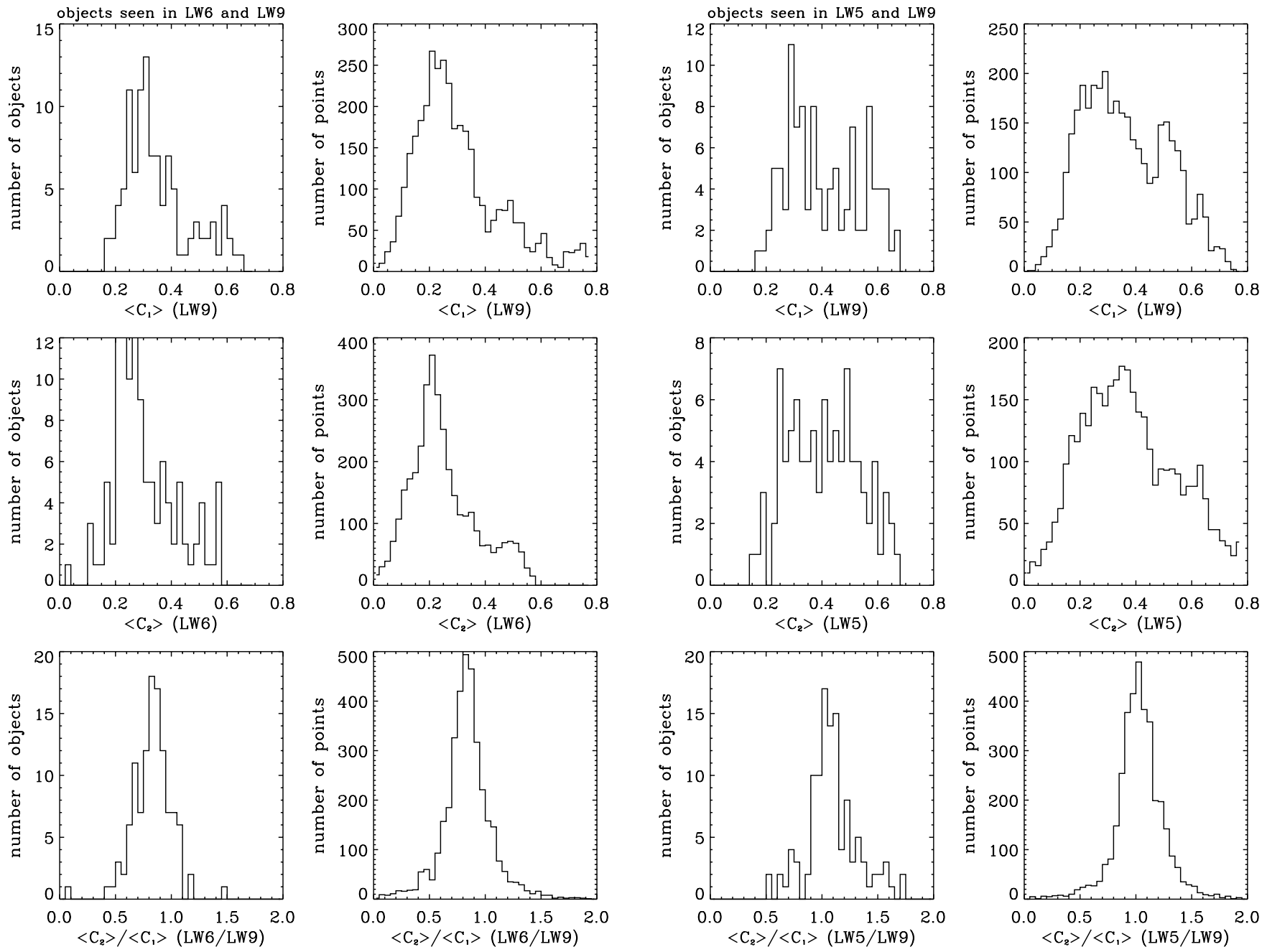

Fig. 21. Same as Fig. 13 for the clouds observed with the filters LW9 and LW6

analysed in the same way as shown in Sects. 4 and 5 for the clouds belonging to the first group.

\subsection{Clouds seen with the LW9 and LW6 filters}

The angular surface of the clouds belonging to the second group is on average larger than the angular surface of the first group clouds (about 7 square arcminutes with the first threshold, 2 for the second and 1 for the third, see Fig. 9). Otherwise the morphology of the second group is similar to the morphology of the first group and is not presented here.

The histograms of $\left\langle C_{1}\right\rangle,\left\langle C_{2}\right\rangle$ and $\left\langle C_{2}\right\rangle /$ $<C_{1}>$ for the second group are displayed in Fig. 21. They are similar to the histograms of the first group (Fig. 13) except that, due to large background intensities (Fig. 19) the distribution of $\left\langle C_{1}>\right.$ and $<C_{2}>$ present larger wings.

The value of the ratio $\left\langle C_{2}>/<C_{1}>\right.$ for the second group is equal to $\simeq 0.85 \pm 0.15$. It is a little higher than the value $0.75 \pm 0.15$ found for the first group, but not inconsistent with this wider band, wider area value.
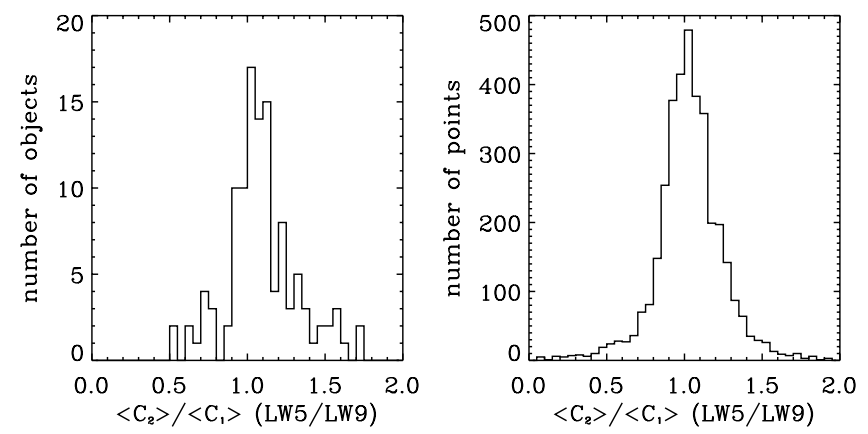

Fig. 22. Same as Fig. 13 for the clouds observed with the filters LW9 and LW5

Several explanations for this higher value will be discussed in the next section.

\subsection{Clouds seen with the LW9 and LW5 filters}

The third group (LW9-LW5) differs more significantly from the first group. The distributions of $\left\langle C_{1}\right\rangle$ and $<C_{2}>$ are broader than for the two previous cases. Both distributions of $\left\langle C_{1}>\right.$ and $\left\langle C_{2}>\right.$ peak around 0.3. The wings are much more important than for the first and the second group. This is due to the larger background intensities (Fig. 19) and likely also to an increase of the clouds opacity. The shape of the distribution of $\left.\left\langle C_{2}\right\rangle /<C_{1}\right\rangle$ is similar to the two previous cases except that it peaks at $\simeq 1.05 \pm 0.15$. Although massive clouds with larger opacity $(\tau=4-5)$ could explain a value of $\left\langle C_{2}\right\rangle /\left\langle C_{1}\right\rangle$ equal to 0.9-1 (see Fig. 14), it cannot explain that most of the points are above 1 except if $\beta_{2} / \beta_{1}$ is significantly larger than 1 . A simple way of achieving such values without changing the colours of the dust emission along the line of sight in an ad-hoc way, is to take extinction along 

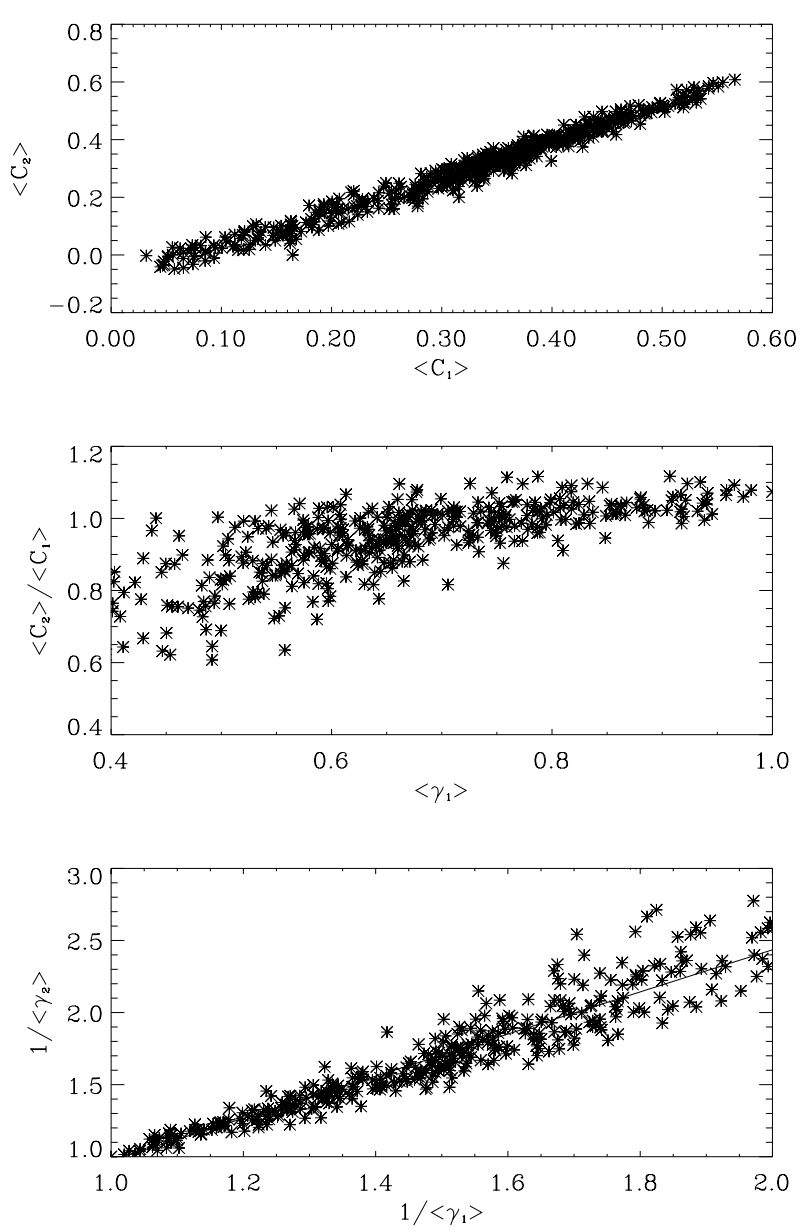

Fig. 23. Same as Fig. 16 for DF- $0.33+0.00$

the line of sight into account. Indeed in the direction of the Galactic Centre opacities up to 1 are expected at $15 \mu \mathrm{m}$.

If we assume that the source function is constant along the line of sight, and that the extinction in front of and behind the cloud are respectively $\tau_{\mathrm{f}}$ and $\tau_{\mathrm{b}}$, the observed intensities write:

$$
\begin{aligned}
I(\tau)= & S\left(\left(1-\exp \left(-\tau_{\mathrm{b}}\right)\right) \exp \left(-\left(\tau_{\mathrm{f}}+\tau\right)\right)\right. \\
& \left.+\left(1-\exp \left(-\tau_{\mathrm{f}}\right)\right)\right)
\end{aligned}
$$

where $S$ is the source function and $\tau$, the cloud opacity. The $\beta_{2} / \beta_{1}$ ratio then writes:

$\frac{\beta_{2}}{\beta_{1}}=\frac{\exp \left(\tau_{\mathrm{b} 2}\right)-1}{\exp \left(\tau_{\mathrm{b} 1}\right)-1} \times \frac{\exp \left(\tau_{\mathrm{b} 1}+\tau_{\mathrm{f} 1}\right)-1}{\exp \left(\tau_{\mathrm{b} 2}+\tau_{\mathrm{f} 2}\right)-1}$.

A $\tau_{2} / \tau_{1}$ opacity ratio different from 1 will result into $\beta_{2} / \beta_{1}$ ratios not equal to 1 , even with the assumption of a uniform source function. Assuming again that $\tau_{2}=0.7 \tau_{1}$, the corresponding order of magnitude for $\tau_{\mathrm{b} 1}=\tau_{\mathrm{f} 1}=0.5$ is 1.1 and 1.2 for $\tau_{\mathrm{b} 1}=\tau_{\mathrm{f} 1}=1$. Thus, the contrast ratio has to be divided by a factor 1.1 to 1.2 in order to correct its value from the diffuse extinction. This leads to a typical value around 0.9 more consistent with first group clouds, although likely to have higher optical depths. This would imply that most clouds of the third group are further away from the Sun, hence really close to the Galactic Centre.
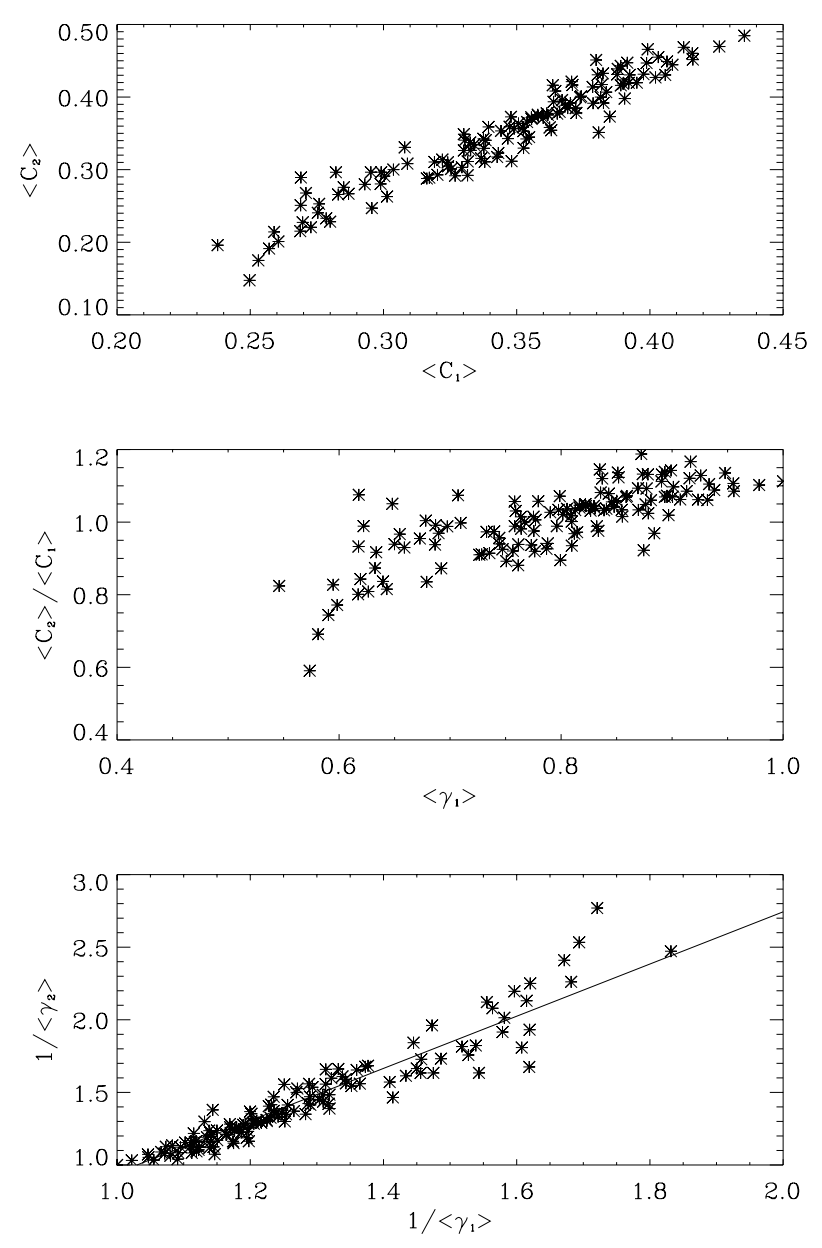

Fig. 24. Same as Fig. 16 for $\mathrm{DF}+0.45+0.23$

Alternative explanations might have been:

- A systematic gradient of the colour ratio at 7 and $15 \mu \mathrm{m}$ near the Galactic Centre that could be due to the star contribution to the background intensity in this region (the ratio $\beta_{2} / \beta_{1}$ becomes significantly different from 1). However this explanation is not supported by the fact that we observe no systematic gradient in the background colour ratio (Fig. 19);

- A variation of $\alpha$ itself resulting from an evolution of the dust properties near the Galactic Centre as for example a gradient of the abundance ratio of graphite and silicate particles which are responsible for the absorption respectively at 7 and $15 \mu \mathrm{m}$. Indeed, the corresponding opacity ratio derived with our model $(\alpha \simeq 0.9)$ is in acceptable agreement with the value obtained from the extinction measurement of Lutz (1998). However, maximum opacity ratios can be derived from the relative variation of $\left\langle C_{2}\right\rangle /\left\langle C_{1}\right\rangle$ within the boundaries of one cloud (see Sect. 5). Two examples are shown (Figs. 23 and 24) which both imply $\alpha \leq 0.7$, irrespective of any additional assumption. This finding contradicts Lutz's (1998) derivation of $\alpha \simeq 0.9$. An equivalent trend is shown by the normalised contrast scatter diagrams, which exhibit slopes around 1.5, inconsistent with $\alpha$ values close to 1 . 
It seems thus convincing that third group clouds (and partly second group) are more massive than inner disk clouds belonging to first group observations. Opacities larger than 4 are indeed obtained for the two examples presented here (slope of the $1 / \gamma_{2}$ vs. $1 / \gamma_{1}$ curve equal to $1.6 \pm 0.2$ for $\mathrm{DF}-0.33+0.00$ and $1.8 \pm 0.2$ for $\mathrm{DF}+0.45+0.23)$.

\section{Conclusions}

In this paper, we used the ISOGAL data (after zodiacal emission subtraction) to measure the variations of the background intensity in the two bands LW2 and LW3 with galactic longitude and latitude.

Using a multiresolution analysis, we have extracted the dark features seen in absorption and studied their morphology and location. Although few data are available at longitudes larger than $40^{\circ}$, the distribution is consistent with the distribution of young components (see also Egan et al. 1998). These dark clouds are condensed cores of galactic giant molecular clouds. Near Infrared and radio follow-ups will refine this view.

We studied the cross-correlation of intensity fluctuations in both 7 and $15 \mu \mathrm{m}$ bands available. A 7 to $15 \mu \mathrm{m}$ contrast ratio of $0.75 \pm 0.15$ has been inferred for the inner disk sample. A simple model translates this ratio into a 7 to $15 \mu \mathrm{m}$ opacity ratio of $0.7 \pm 0.1$ in good agreement with the extinction curve calculated by Draine \& Lee (1984) but not consistent with the value obtained by Lutz (1998). For two values (0.6 and 0.7) of the opacity ratio, we derived the maximum opacity reached for six of these clouds. Typical values are $\tau \simeq 1-4$ leading to $\mathrm{H}$ column densities ranging from $1-410^{23} \mathrm{~cm}^{-2}$ for solar neighbourhood conditions.

The contrast ratio for the clouds seen with the narrower filters LW6 and LW9 and located near the Galactic Centre $\left(|l|<1^{\circ}, 0.2^{\circ}<|b|<0.4^{\circ}\right)$ is $0.85 \pm 0.15$ whereas it is $1.05 \pm 0.15$ for the clouds seen with the filters LW5 and LW9 and located at lower latitude $\left(|l|<1^{\circ},|b|<0.2^{\circ}\right)$. This last result is straightforwardly accounted for by diffuse extinction along the line of sight and the fact that the clouds near the Galactic Centre are more massive than clouds of the inner disk. These data are not consistent with Lutz's (1998) finding of a significantly different mid Infrared extinction curve in the Galactic Centre area.
Acknowledgements. The authors wish to thank Bill Reach for his decisive help in zodiacal emission estimates and Albert Bijaoui, Eric Slezac and Benoit Vandame for providing the MVM software. This work much benefitted from the involvement of the ISOCAM instrument team and of the ISOGAL team. Let them all be warmly thanked. We also thank Patrick Boissé for several stimulating discussions.

\section{References}

Abergel, A., Bernard, J. P., Boulanger, F., et al. 1996, A\&A, 315, L329

Abergel, A., Bernard, J. P., Boulanger, F., et al. 1998, in The Universe as seen by ISO, ESA

Barnard, E. 1919, ApJ, 49, 1

Bloemen, J., Deul, E., \& Thaddeus, P. 1990, A\&A, 233, 437

Bok, B., \& Reilly, E. 1947, ApJ, 105, 255

Boulanger, F., \& Pérault, M. 1988, ApJ, 330, 964

Carey, S. J., Clark, F. O., Egan, M. P., et al. 1998, ApJ, 508, 721

Chandrasekhar, S., \& Münch, G. 1952, ApJ, 115, 103

Cohen, R., Cong, H., Dame, T., \& Thaddeus, P. 1980, ApJ, 239,53

Draine, B., \& Lee, H. 1984, ApJ, 285, 89

Egan, M. P., Shipman, R. F., Price, S. D., et al. 1998, ApJ, 494, L199

Kelsall, T., Weiland, J., Franz, B., et al. 1998, ApJ, 508, 44

Lis, D., \& Menten, K. 1998, ApJ, 507, 794

Lis, D., Li, Y., Dowell, C., \& Menten, K. 1998, in The universe as seen by ISO, ESA

Lutz, D., Feuchtgruber, H., Genzel, R., et al. 1996, A\&A, 315, L269

Lutz, D. 1998, in The Universe as seen by ISO, ESA

Lynds, B. T. 1962, ApJ, S, 7, 1

Omont, A., Ganesh, S., Alard, C., et al. 1999, A\&A, 348, 755

Pérault, M., 1987, Ph.D. dissertation Structure et évolution des nuages moléculaires, Université de Paris VII

Pérault, M., Omont, A., Simon, G., et al. 1996, A\&A, 315, L165

Price, S. D. 1981, ApJ, 86, 193

Rué, F., \& Bijaoui, A. 1997, Exper. Astron., 7, 129

Sanders, D., Solomon, P., \& Scoville, N. 1984, ApJ, 276, 182

Sodroski, T., Dwek, E., Hauser, M., \& Kerr, F. 1989, ApJ, 336, 762

Sodroski, T., Bennett, C., Boggess, N., Dwek, E., \& Franz, B. 1994, ApJ, 428, 638

Solomon, S., Sanders, D., Scoville, N. 1979, ApJ, 232, L89

Starck, J. L., Abergel, A., Aussel, H., Sauvage, M., et al. 1999, A\&AS, 134, 135

Teyssier, D., Pérault, M., \& Hennebelle, P. 2001, in preparation 